\title{
Inversion of bilateral basic hypergeometric series
}

\author{
Michael Schlosser* \\ Institut für Mathematik der Universität Wien \\ Strudlhofgasse 4, A-1090 Wien, Austria \\ schlosse@ap.univie.ac.at \\ Submitted: Jun 5, 2002; Accepted: Jan 8, 2003; Published: Mar 18, 2003 \\ MR Subject Classifications: 33D15, 15A09
}

\begin{abstract}
We present a new matrix inverse with applications in the theory of bilateral basic hypergeometric series. Our matrix inversion result is directly extracted from an instance of Bailey's very-well-poised ${ }_{6} \psi_{6}$ summation theorem, and involves two infinite matrices which are not lower-triangular. We combine our bilateral matrix inverse with known basic hypergeometric summation theorems to derive, via inverse relations, several new identities for bilateral basic hypergeometric series.
\end{abstract}

\section{Introduction}

Bailey's [10, Eq. (4.7)] very-well-poised ${ }_{6} \psi_{6}$ summation formula,

$$
\begin{array}{r}
{ }_{6} \psi_{6}\left[\begin{array}{r}
q \sqrt{a},-q \sqrt{a}, b, c, d, e \\
\sqrt{a},-\sqrt{a}, a q / b, a q / c, a q / d, a q / e
\end{array} ;, \frac{a^{2} q}{b c d e}\right] \\
=\frac{(q, a q, q / a, a q / b c, a q / b d, a q / b e, a q / c d, a q / c e, a q / d e ; q)_{\infty}}{\left(a q / b, a q / c, a q / d, a q / e, q / b, q / c, q / d, q / e, a^{2} q / b c d e ; q\right)_{\infty}}
\end{array}
$$

where $\left|a^{2} q / b c d e\right|<1$ (cf. [19, Eq. (5.3.1)]), stands on the top of the classical hierarchy of summation theorems for bilateral basic hypergeometric series. It contains many important identities as special cases, among them Jacobi's triple product identity, the $q$-Pfaff-Saalschütz summation, and the $q$-binomial theorem, to name just a few. Various applications of Bailey's ${ }_{6} \psi_{6}$ summation exist in number theory (see Andrews [4, pp. 461-468]) and in special functions (see, e.g., Ismail and Masson [23]). A combinatorial (partition theoretic) application of Bailey's ${ }_{6} \psi_{6}$ summation formula was recently revealed in remarkable work of Alladi, Andrews, and Berkovich [2].

\footnotetext{
${ }^{*}$ Supported by an APART grant of the Austrian Academy of Sciences
} 
Different proofs of (1.1) are known. A very elegant proof using analytic continuation was given by Askey and Ismail [9]. For an elementary proof using manipulations of series, see Schlosser [38].

In addition to Bailey's ${ }_{6} \psi_{6}$ summation formula, there is a significant number of other important summation and transformation theorems for basic hypergeometric series (cf. [19]). Basic hypergeometric series (and, more generally, $q$-series) have various applications in combinatorics, number theory, representation theory, statistics, and physics, see Andrews [4],[6]. For a general account of the importance of basic hypergeometric series in the theory of special functions see Andrews, Askey, and Roy [8].

Various techniques have been employed for the study of basic hypergeometric series. A fundamental approach is to start with simple identities and build up the theory by successively deriving more complicated identities. The heart piece of this method is the "Bailey transform", a simple but efficient interchange of summation argument. The Bailey transform is even more powerful if it is combined with a specific summation theorem, in which case it becomes a "Bailey lemma" (see Andrews [6],[7]). Starting with an identity, the Bailey lemma generates an infinite chain (or lattice) of identities, a so-called "Bailey chain", or more general, a "Bailey lattice" (see [6] and [1]).

Another important tool for proving or deriving identities is using "inverse relations", which are an immediate consequence of matrix inversions. By this method, the proof of a given identity may be reduced to the proof of another "dual" identity. On the other hand, given a known identity, by applying inverse relations a possibly new identity may be derived.

Matrix inversion and Bailey lemma are not unrelated tools for deriving identities. Already Andrews [5] had observed that the specific application of the Bailey transform in the classical Bailey lemma is equivalent to an explicit matrix inversion result. Important matrix inversions have been found by Gould and Hsu [21], Carlitz [14], Gessel and Stanton [20], Bressoud [13], Al-Salam and Verma [3], Gasper [17], Krattenthaler [26], and Warnaar [41]. Similar results in higher dimensions (related to multiple series) have been obtained by Chu [15], Milne [30], Lilly and Milne [28], Bhatnagar and Milne [12], Schlosser [35],[36], and Krattenthaler and Schlosser [27].

In this paper, we provide yet another explicit pair of infinite matrices being inverses of each other. The difference from all the previously mentioned matrix inverses is that our new result involves two infinite matrices which are not necessarily lower-triangular, i.e., all their entries may be non-zero. The corresponding orthogonality relations are infinite convergent sums. Our new "bilateral matrix inverse" in Theorem 3.1 is directly extracted from an instance of Bailey's very-well-poised ${ }_{6} \psi_{6}$ summation (1.1) and extends Bressoud's [13] matrix inverse (which involves lower-triangular matrices) by an additional free parameter.

After a short introduction to basic hypergeometric series and inverse relations in Section 2, we state and prove our main result, a "bilateral matrix inverse", in Section 3. In Section 4, we combine our new matix inverse with basic hypergeometric summation theorems to derive, via inverse relations, new bilateral summation theorems. Finally, in Section 5, we use our newly derived summations from Section 4 to deduce by elementary 
manipulations of sums further bilateral series identities.

In a forthcoming paper, we apply part of the current analysis to multiple sums. In particular, by appropriately specializing Gustafson's [22] $A_{r}$ and $C_{r}{ }_{6} \psi_{6}$ summations, we derive multidimensional extensions of our bilateral matrix inverse in Theorem 3.1, and deduce some multilateral summations as applications.

We are currently preparing another article which features a new bilateral Bailey lemma, based on our new bilateral matrix inverse in Theorem 3.1, combined with Bailey's [11, Eq. (3.3.)] nonterminating ${ }_{8} \phi_{7}$ summation. This bilateral Bailey lemma is different from (and does not specialize to) the Bailey lemmas considered in [6] or [7].

This paper has been typeset while the author was visiting Northwestern University in Evanston, Illinois, for the Spring Quarter 2002. The author wishes to acknowledge the positive research atmosphere experienced there. We are in particular thankful for stimulating discussions with George Gasper.

\section{Preliminaries}

\subsection{Notation and basic hypergeometric series}

Here we recall some standard notation for $q$-series, and basic hypergeometric series (cf. [19]).

Let $q$ be a complex number such that $0<|q|<1$. We define the $q$-shifted factorial for all integers $k$ by

$$
(a ; q)_{\infty}:=\prod_{j=0}^{\infty}\left(1-a q^{j}\right) \quad \text { and } \quad(a ; q)_{k}:=\frac{(a ; q)_{\infty}}{\left(a q^{k} ; q\right)_{\infty}}
$$

For brevity, we employ the condensed notation

$$
\left(a_{1}, \ldots, a_{m} ; q\right)_{k} \equiv\left(a_{1} ; q\right)_{k} \ldots\left(a_{m} ; q\right)_{k}
$$

where $k$ is an integer or infinity. Further, we utilize

$$
{ }_{s} \phi_{s-1}\left[\begin{array}{c}
a_{1}, a_{2}, \ldots, a_{s} \\
b_{1}, b_{2}, \ldots, b_{s-1}
\end{array} ; q, z\right]:=\sum_{k=0}^{\infty} \frac{\left(a_{1}, a_{2}, \ldots, a_{s} ; q\right)_{k}}{\left(q, b_{1}, \ldots, b_{s-1} ; q\right)_{k}} z^{k}
$$

and

$$
{ }_{s} \psi_{s}\left[\begin{array}{l}
a_{1}, a_{2}, \ldots, a_{s} \\
b_{1}, b_{2}, \ldots, b_{s}
\end{array} ; q, z\right]:=\sum_{k=-\infty}^{\infty} \frac{\left(a_{1}, a_{2}, \ldots, a_{s} ; q\right)_{k}}{\left(b_{1}, b_{2}, \ldots, b_{s} ; q\right)_{k}} z^{k},
$$

to denote the basic hypergeometric ${ }_{s} \phi_{s-1}$ series, and the bilateral basic hypergeometric ${ }_{s} \psi_{s}$ series, respectively. In $(2.1)$ or $(2.2), a_{1}, \ldots, a_{s}$ are called the upper parameters, $b_{1}, \ldots, b_{s}$ the lower parameters, $z$ is the argument, and $q$ the base of the series. See $[19$, p. 25 and p. 125] for the criteria of when these series terminate, or, if not, when they converge.

The classical theory of basic hypergeometric series contains numerous summation and transformation formulae involving ${ }_{s} \phi_{s-1}$ or ${ }_{s} \psi_{s}$ series. Many of these summation theorems 
require that the parameters satisfy the condition of being either balanced and/or very-wellpoised. An ${ }_{s} \phi_{s-1}$ basic hypergeometric series is called balanced if $b_{1} \cdots b_{s-1}=a_{1} \cdots a_{s} q$ and $z=q$. An ${ }_{s} \phi_{s-1}$ series is well-poised if $a_{1} q=a_{2} b_{1}=\cdots=a_{s} b_{s-1}$. An ${ }_{s} \phi_{s-1}$ basic hypergeometric series is called very-well-poised if it is well-poised and if $a_{2}=-a_{3}=q \sqrt{a_{1}}$. Note that the factor

$$
\frac{1-a_{1} q^{2 k}}{1-a_{1}}
$$

appears in a very-well-poised series. The parameter $a_{1}$ is usually referred to as the special parameter of such a series. Similarly, a bilateral ${ }_{s} \psi_{s}$ basic hypergeometric series is wellpoised if $a_{1} b_{1}=a_{2} b_{2} \cdots=a_{s} b_{s}$ and very-well-poised if, in addition, $a_{1}=-a_{2}=q b_{1}=$ $-q b_{2}$. Further, we call a bilateral ${ }_{s} \psi_{s}$ basic hypergeometric series balanced if $b_{1} \cdots b_{s}=$ $a_{1} \cdots a_{s} q^{2}$ and $z=q$.

A standard reference for basic hypergeometric series is Gasper and Rahman's text [19]. In our computations in the subsequent sections we frequently use some elementary identities of $q$-shifted factorials, listed in [19, Appendix I].

In the following we display some summation theorems which we utilize in Sections 4 and 5 .

One of the most important theorems in the theory of basic hypergeometric series is Jackson's [24] terminating very-well-poised balanced ${ }_{8} \phi_{7}$ summation (cf. [19, Eq. (2.6.2)]):

$$
\begin{aligned}
&{ }_{8} \phi_{7}\left[\begin{array}{c}
a, q \sqrt{a},-q \sqrt{a}, b, c, d, a^{2} q^{1+n} / b c d, q^{-n} \\
\sqrt{a},-\sqrt{a}, a q / b, a q / c, a q / d, b c d q^{-n} / a, a q^{1+n}
\end{array} ;, q\right] \\
&= \frac{(a q, a q / b c, a q / b d, a q / c d ; q)_{n}}{(a q / b, a q / c, a q / d, a q / b c d ; q)_{n}} .
\end{aligned}
$$

A less well known but nevertheless very useful identity is the following very-well-poised ${ }_{8} \phi_{7}$ summation:

$$
\begin{aligned}
{ }_{8} \phi_{7}\left[\begin{array}{r}
\lambda, q \sqrt{\lambda},-q \sqrt{\lambda}, a, b, c,-c, \lambda q / c^{2} \\
\sqrt{\lambda},-\sqrt{\lambda}, \lambda q / a, \lambda q / b, \lambda q / c,-\lambda q / c, c^{2}
\end{array} ; q,-\frac{\lambda q}{a b}\right] \\
=\frac{\left(\lambda q, c^{2} / \lambda ; q\right)_{\infty}\left(a q, b q, c^{2} q / a, c^{2} q / b ; q^{2}\right)_{\infty}}{(\lambda q / a, \lambda q / b ; q)_{\infty}\left(q, a b q, c^{2} q, c^{2} q / a b ; q^{2}\right)_{\infty}}
\end{aligned}
$$

provided $|\lambda q / a b|<1$, where $\lambda=-c \sqrt{a b / q}$.

The ${ }_{8} \phi_{7}$ summation formula in (2.4) is a $q$-analogue of a ${ }_{3} F_{2}$ summation due to Whipple [43] (but commonly attributed to Watson [42] who gave that ${ }_{3} F_{2}$ summation for the case when the series is terminating).

A bilateral summation even (slightly) more general than the ${ }_{6} \psi_{6}$ sum in (1.1) is H. S. Shukla's [40] very-well-poised ${ }_{8} \psi_{8}$ summation:

$$
\begin{aligned}
& { }_{8} \psi_{8}\left[\begin{array}{c}
q \sqrt{a},-q \sqrt{a}, b, c, d, e, f, a q^{2} / f \\
\sqrt{a},-\sqrt{a}, a q / b, a q / c, a q / d, a q / e, a q / f, f / q
\end{array} ; q, \frac{a^{2}}{b c d e}\right] \\
& =\left(1-\frac{(1-b c / a)(1-b d / a)(1-b e / a)}{(1-b q / f)(1-b f / a q)\left(1-b c d e / a^{2}\right)}\right) \frac{(1-f / b q)(1-b f / a q)}{(1-f / a q)(1-f / q)}
\end{aligned}
$$




$$
\times \frac{(q, a q, q / a, a q / b c, a q / b d, a q / b e, a q / c d, a q / c e, a q / d e ; q)_{\infty}}{\left(a q / b, a q / c, a q / d, a q / e, q / b, q / c, q / d, q / e, a^{2} q / b c d e ; q\right)_{\infty}},
$$

where $\left|a^{2} / b c d e\right|<1$. Note that (2.5) reduces to (1.1) if $f \rightarrow 0$ or $f \rightarrow \infty$. For a generalization of (2.5), see (4.10).

We will use the summations (2.3), (2.4), and (2.5) in Section 4 to derive new bilateral summation theorems.

\section{$2.2 \quad$ Inverse relations}

Let $\mathbb{Z}$ denote the set of integers. In the following, we consider infinite matrices $\left(f_{n k}\right)_{n, k \in \mathbb{Z}}$ and $\left(g_{n k}\right)_{n, k \in \mathbb{Z}}$, and infinite sequences $\left(a_{n}\right)_{n \in \mathbb{Z}}$ and $\left(b_{n}\right)_{n \in \mathbb{Z}}$.

We say that the infinite matrices $\left(f_{n k}\right)_{n, k \in \mathbb{Z}}$ and $\left(g_{k l}\right)_{k, l \in \mathbb{Z}}$ are inverses of each other if and only if the following orthogonality relation holds:

$$
\sum_{k \in \mathbb{Z}} f_{n k} g_{k l}=\delta_{n l} \quad \text { for all } \quad n, l \in \mathbb{Z} .
$$

Clearly, since inverse matrices commute, we also then have

$$
\sum_{l \in \mathbb{Z}} g_{k l} f_{l j}=\delta_{k j} \quad \text { for all } \quad k, j \in \mathbb{Z} .
$$

Note that in (2.6) and (2.7) we are not requiring that the infinite matrices are lowertriangular. If they were, the summations on the left hand sides of (2.6) and (2.7) would be in fact finite sums. In the general case, the sums will be infinite. If the summands of the infinite series involve complex numbers, we require suitable convergence conditions to hold (such as absolute convergence; for interchanging double sums we also need uniform convergence).

It is immediate from the orthogonality relations (2.6) and (2.7) that the following inverse relations hold: Let $\left(f_{n k}\right)_{n, k \in \mathbb{Z}}$ and $\left(g_{k l}\right)_{k, l \in \mathbb{Z}}$ be infinite matrices being inverses of each other. Then

$$
\sum_{k \in \mathbb{Z}} f_{n k} a_{k}=b_{n} \quad \text { for all } n,
$$

if and only if

$$
\sum_{l \in \mathbb{Z}} g_{k l} b_{l}=a_{k} \quad \text { for all } k .
$$

The other variant of inverse relations, which may be called "rotated inversion" (see also Riordan [31]), reads as follows: Let $\left(f_{n k}\right)_{n, k \in \mathbb{Z}}$ and $\left(g_{k l}\right)_{k, l \in \mathbb{Z}}$ be infinite matrices being inverses of each other. Then

$$
\sum_{n \in \mathbb{Z}} f_{n k} a_{n}=b_{k} \quad \text { for all } k,
$$

if and only if

$$
\sum_{k \in \mathbb{Z}} g_{k l} b_{k}=a_{l} \quad \text { for all } l .
$$


We also note here that if the considered sequences involve complex numbers we need suitable convergence conditions for the above pairs of inverse relations $(2.8) /(2.9)$ and $(2.10) /(2.11)$ to hold.

Inverse relations are a powerful tool for proving or deriving identities. For instance, given an identity in the form (2.11), we can immediately deduce (2.10), which may possibly be a new identity. It is exactly this variant of inverse relations which we will utilize in Section 4 to derive new summation theorems for bilateral series.

\section{A new bilateral matrix inverse}

We now present our main result, an explicit pair of inverse infinite matrices which are not lower-triangular.

Theorem 3.1 Let $a, b$, and $c$ be indeterminates. The infinite matrices $\left(f_{n k}\right)_{n, k \in \mathbb{Z}}$ and $\left(g_{k l}\right)_{k, l \in \mathbb{Z}}$ are inverses of each other where

$$
\begin{aligned}
f_{n k}=\frac{(a q / b, b q / a, a q / c, c q / a, b q, q / b, c q, q / c ; q)_{\infty}}{(q, q, a q, q / a, a q / b c, b c q / a, c q / b, b q / c ; q)_{\infty}} & \\
\times & \times \frac{\left(1-b c q^{2 n} / a\right)}{(1-b c / a)} \frac{(b ; q)_{n+k}(a / c ; q)_{k-n}}{(c q ; q)_{n+k}(a q / b ; q)_{k-n}}
\end{aligned}
$$

and

$$
g_{k l}=\frac{\left(1-a q^{2 k}\right)}{(1-a)} \frac{(c ; q)_{k+l}(a / b ; q)_{k-l}}{(b q ; q)_{k+l}(a q / c ; q)_{k-l}} q^{k-l}
$$

Remark 3.2 If we let $c \rightarrow a$ in Theorem 3.1, we obtain a matrix inverse found by Bressoud [13] which he directly extracted from the terminating very-well-poised ${ }_{6} \phi_{5}$ summation (a special case of (1.1)). If, after letting $c \rightarrow a$, we additionally let $a \rightarrow 0$, we obtain Andrews' [5, Lemma 3] "Bailey transform matrices", a matrix inversion underlying the powerful Bailey lemma.

Proof of Theorem 3.1. We show that the inverse matrices $(3.1) /(3.2)$ satisfy the orthogonality relation (2.6). Writing out the sum $\sum_{k \in \mathbb{Z}} f_{n k} g_{k l}$ with the above choices of $f_{n k}$ and $g_{k l}$ we observe that the series can be summed by an application of Bailey's verywell-poised ${ }_{6} \psi_{6}$ summation (1.1). The specializations needed there are $b \mapsto b q^{n}, c \mapsto c q^{l}$, $d \mapsto a q^{-l} / b$, and $e \mapsto a q^{-n} / c$. Bailey's formula then gives us a product containing the factors $\left(q^{1-n+l}, q^{1+n-l} ; q\right)_{\infty}$. Since

$$
\left(q^{1-n+l}, q^{1+n-l} ; q\right)_{\infty}=0
$$

for all integers $n$ and $l$ with $n \neq l$, we can simplify the product (setting $n=l$, the only non-zero case) and readily determine that the sum indeed boils down to $\delta_{n l}$. The details are as follows: 


$$
\begin{gathered}
\sum_{k \in \mathbb{Z}} f_{n k} g_{k l}=\sum_{k=-\infty}^{\infty} \frac{(a q / b, b q / a, a q / c, c q / a, b q, q / b, c q, q / c ; q)_{\infty}}{(q, q, a q, q / a, a q / b c, b c q / a, c q / b, b q / c ; q)_{\infty}} \\
\times \frac{\left(1-b c q^{2 n} / a\right)}{(1-b c / a)} \frac{(b ; q)_{n+k}(a / c ; q)_{k-n}}{(c q ; q)_{n+k}(a q / b ; q)_{k-n}} \frac{\left(1-a q^{2 k}\right)}{(1-a)} \frac{(c ; q)_{k+l}(a / b ; q)_{k-l}}{(b q ; q)_{k+l}(a q / c ; q)_{k-l}} q^{k-l} \\
=\frac{(a q / b, b q / a, a q / c, c q / a, b q, q / b, c q, q / c ; q)_{\infty}}{(q, q, a q, q / a, a q / b c, b c q / a, c q / b, b q / c ; q)_{\infty}} \\
\times \frac{\left(1-b c q^{2 n} / a\right)}{(1-b c / a)} \frac{(b ; q)_{n}(a / c ; q)_{-n}(c ; q)_{l}(a / b ; q)_{-l}}{(c q ; q)_{n}(a q / b ; q)_{-n}(b q ; q)_{l}(a q / c ; q)_{-l}} q^{-l} \\
\times \sum_{k=-\infty}^{\infty} \frac{\left(1-a q^{2 k}\right)}{(1-a)} \frac{\left(b q^{n}, a q^{-n} / c, c q^{l}, a q^{-l} / b ; q\right)_{k}}{\left(a q^{1-n} / b, c q^{1+n}, a q^{1-l} / c, b q^{1+l} ; q\right)_{k}} q^{k} \\
=\frac{(a q / b, b q / a, a q / c, c q / a, b q, q / b, c q, q / c ; q)_{\infty}}{(q, q, a q, q / a, a q / b c, b c q / a, c q / b, b q / c ; q)_{\infty}} \\
\times \frac{\left(1-b c q^{2 n} / a\right)}{(1-b c / a)} \frac{(b ; q)_{n}(a / c ; q)_{-n}(c ; q)_{l}(a / b ; q)_{-l}}{(c q ; q)_{n}(a q / b ; q)_{-n}(b q ; q)_{l}(a q / c ; q)_{-l}} q^{-l} \\
\times \frac{\left(q, a q, q / a, c q / b, a q^{1-n-l} / b c, q^{1-n+l}, q^{1+n-l}, b c q^{1+n+l} / a, b q / c ; q\right)_{\infty}}{\left(a q^{1-n} / b, c q^{1+n}, a q^{1-l} / c, b q^{1+l}, q^{1-n} / b, c q^{1+n} / a, q^{1-l} / c, b q^{1+l} / a, q ; q\right)_{\infty}}
\end{gathered}
$$

where we have been using some elementary identities involving $q$-shifted factorials (cf. [19, Appendix I]).

Observe that the dual orthogonality relation (2.7) of the matrices (3.1)/(3.2) is established by a similar instance of Bailey's very-well-poised ${ }_{6} \psi_{6}$ summation (where the argument is again $q$ ). In particular, both series in (2.6) and in (2.7) converge absolutely for $|q|<1$.

Remark 3.3 We do not need the full ${ }_{6} \psi_{6}$ summation (1.1) to prove the above orthogonality relation(s). All we really need is the very-well-poised balanced ${ }_{6} \psi_{6}$ summation,

$$
\begin{aligned}
\sum_{k=-\infty}^{\infty} \frac{\left(1-a q^{2 k}\right)}{(1-a)} \frac{\left(b, c, d, a^{2} / b c d ; q\right)_{k}}{(a q / b, a q / c, a q / d, b c d q / a ; q)_{k}} q^{k} \\
=\frac{(a q, q / a, a q / b c, b c q / a, a q / b d, b d q / a, c d q / a, a q / c d ; q)_{\infty}}{\left(a q / b, a q / c, a q / d, q / b, q / c, q / d, b c d q / a, b c d q / a^{2} ; q\right)_{\infty}}
\end{aligned}
$$

which is just a special case of Bailey's very-well-poised ${ }_{6} \psi_{6}$ summation (1.1). Usually, behind a particular orthogonality relation there is a more general summation theorem. In case of finite sums, this summation theorem can often be proved by a simple telescoping argument. A similar situation happens here with (3.3). Gasper and Rahman [18] showed that the indefinite bibasic sum 


$$
\begin{gathered}
\sum_{k=-m}^{n} \frac{\left(1-a d p^{k} q^{k}\right)\left(1-b p^{k} / d q^{k}\right)}{(1-a d)(1-b / d)} \frac{(a, b ; p)_{k}\left(c, a d^{2} / b c ; q\right)_{k}}{(d q, a d q / b ; q)_{k}(a d p / c, b c p / d ; p)_{k}} q^{k} \\
=\frac{(1-a)(1-b)(1-c)\left(1-a d^{2} / b c\right)}{d(1-a d)(1-b / d)(1-c / d)(1-a d / b c)} \\
\times\left(\frac{(a p, b p ; p)_{n}\left(c q, a d^{2} q / b c ; q\right)_{n}}{(d q, a d q / b ; q)_{n}(a d p / c, b c p / d ; p)_{n}}-\frac{(c / a d, d / b c ; p)_{m+1}(1 / d, b / a d ; q)_{m+1}}{\left(1 / c, b c / a d^{2} ; q\right)_{m+1}(1 / a, 1 / b ; p)_{m+1}}\right)
\end{gathered}
$$

telescopes. Replacing $a$ and $d$, respectively, by $d$ and $a / d$, letting $n, m \rightarrow \infty$, and setting $p=q$, the above summation reduces to

$$
\begin{aligned}
& \sum_{k=-\infty}^{\infty} \frac{\left(1-a q^{2 k}\right)}{(1-a)} \frac{\left(b, c, d, a^{2} / b c d ; q\right)_{k}}{(a q / b, a q / c, a q / d, b c d q / a ; q)_{k}} q^{k} \\
&=\frac{(1-b)(1-c)(1-d)\left(1-b c d / a^{2}\right)}{(1-a)(1-b d / a)(1-c d / a)(1-b c / a)} \\
& \times\left(\frac{\left(b q, c q, d q, a^{2} q / b c d ; q\right)_{\infty}}{(a q / b, a q / c, a q / d, b c d q / a ; q)_{\infty}}-\frac{(b / a, c / a, d / a, a / b c d ; q)_{\infty}}{\left(1 / b, 1 / c, 1 / d, b c d / a^{2} ; q\right)_{\infty}}\right) .
\end{aligned}
$$

Now, (miraculously) the right hand side of (3.4) can be transformed into the right hand side of (3.3) using a theta function identity of Weierstrass [44, p. 451, Example 5] (also cf. [19, Ex. 5.21]).

\section{Some applications}

Here we combine the matrix inverse of Theorem 3.1 with specific summation theorems to derive, via inverse relations, new identites for bilateral basic hypergeometric series. For convenience, we only use the (rotated) inverse relations $(2.10) /(2.11)$, but because of the symmetric structure of our inverse matrices we could as well also employ the inverse relations $(2.8) /(2.9)$ to obtain equivalent results.

In our first application of Theorem 3.1 we apply inverse relations to Jackson's terminating very-well-poised balanced ${ }_{8} \phi_{7}$ summation, and obtain a summation for a particular very-well-poised balanced ${ }_{8} \psi_{8}$ series, see Theorem 4.1. In our second application we apply inverse relations to the nonterminating ${ }_{8} \phi_{7}$ summation in (2.4). This leads us to a new bilateral quadratic summation, see Theorem 4.2. Whereas in our first two applications of Theorem 3.1 we invert a terminating summation, and a nonterminating unilateral summation, respectively, our third application of Theorem 3.1 involves inverting a genuine bilateral summation theorem. It turns out that if we invert the ${ }_{6} \psi_{6}$ summation theorem (1.1), we again end up with (1.1). (We may also invert Rogers' [32, p. 29, second eq.] nonterminating ${ }_{6} \phi_{5}$ summation (cf. [19, Eq. (2.7.1)]) which leads to a summation for a ${ }_{6} \psi_{6}$ series. Unfortunately the ${ }_{6} \psi_{6}$ summation obtained in this way is not as general as (1.1).) In order to derive something new, we climb up higher in the hierarchy of bilateral basic hypergeometric series and invert Shukla's very-well-poised ${ }_{8} \psi_{8}$ summation (2.5). The result is Theorem 4.3 . 
We start with our first application. Using some elementary identities for $q$-shifted factorials on the right hand side, we may deduce from Jackson's terminating very-wellpoised balanced ${ }_{8} \phi_{7}$ summation (2.3) the identity

$$
\begin{array}{r}
{ }_{8} \phi_{7}\left[\begin{array}{r}
a, q \sqrt{a},-q \sqrt{a}, d, c q^{l}, a q^{-l} / b, a b q^{1+N} / c d, q^{-N} \\
\sqrt{a},-\sqrt{a}, a q / d, a q^{1-l} / c, b q^{1+l}, c d q^{-N} / b, a q^{1+N}
\end{array} q, q\right] \\
=\frac{(a q, a q / c d, b q / c, b q / d ; q)_{N}}{(b q / c d, b q, a q / d, a q / c ; q)_{N}} \frac{\left(b q, b q^{1+N} / d, c d / a, c q^{-N} / a ; q\right)_{l}}{\left(c / a, c d q^{-N} / a, b q / d, b q^{1+N} ; q\right)_{l}}
\end{array}
$$

where $N$ is a nonnegative integer. Note that we have introduced an additional integer $l$ in the above ${ }_{8} \phi_{7}$ summation. We can rewrite (4.1) as

$$
\begin{aligned}
\sum_{k \in \mathbb{Z}} \underbrace{\frac{\left(1-a q^{2 k}\right)}{(1-a)} \frac{(c ; q)_{k+l}(a / b ; q)_{k-l}}{(b q ; q)_{k+l}(a q / c ; q)_{k-l}} q^{k-l}}_{g_{k l}} \underbrace{\frac{\left(a, d, a b q^{1+N} / c d, q^{-N} ; q\right)_{k}}{\left(q, a q / d, c d q^{-N} / b, a q^{1+N} ; q\right)_{k}}}_{b_{k}} \\
=\underbrace{\frac{(a q, a q / c d, b q / c, b q / d ; q)_{N}}{(b q / c d, b q, a q / d, a q / c ; q)_{N}} \frac{\left(b q^{1+N} / d, c, c d / a, c q^{-N} / a ; q\right)_{l}}{\left(c d q^{-N} / a, b q / a, b q / d, b q^{1+N} ; q\right)_{l}}\left(\frac{b}{c}\right)^{l}}_{a_{l}},
\end{aligned}
$$

by which we have established (2.11) for the above choices of $a_{l}$ and $b_{k}$. By virtue of the matrix inversion in Theorem 3.1 and the equivalence of (2.10) and (2.11), we immediately deduce from (4.2) the following inverse relation:

$$
\begin{gathered}
\sum_{n \in \mathbb{Z}} \underbrace{\frac{(a q / b, b q / a, a q / c, c q / a, b q, q / b, c q, q / c ; q)_{\infty}\left(1-b c q^{2 n} / a\right)(b ; q)_{n+k}(a / c ; q)_{k-n}}{(q, q, a q, q / a, a q / b c, b c q / a, c q / b, b q / c ; q)_{\infty}(1-b c / a)(c q ; q)_{n+k}(a q / b ; q)_{k-n}}}_{f_{n k}} \\
\times \underbrace{\frac{(a q, a q / c d, b q / c, b q / d ; q)_{N}}{(b q / c d, b q, a q / d, a q / c ; q)_{N}} \frac{\left(b q^{1+N} / d, c, c d / a, c q^{-N} / a ; q\right)_{n}}{\left(c d q^{-N} / a, b q / a, b q / d, b q^{1+N} ; q\right)_{n}}\left(\frac{b}{c}\right)^{n}}_{a_{n}} \\
=\underbrace{\frac{\left(a, d, a b q^{1+N} / c d, q^{-N} ; q\right)_{k}}{\left(q, a q / d, c d q^{-N} / b, a q^{1+N} ; q\right)_{k}}}_{b_{k}} .
\end{gathered}
$$

Now, after substitution of variables (simultaneously $a \mapsto c d / a, b \mapsto d$, and $d \mapsto b d / a$ ), and moving some factors to the other side, we obtain the following very-well-poised balanced ${ }_{8} \psi_{8}$ summation:

Theorem 4.1 Let $a, b, c$, and $d$ be indeterminates, let $k$ be an arbitrary integer and $N$ a nonnegative integer. Then

$$
{ }_{8} \psi_{8}\left[\begin{array}{c}
q \sqrt{a},-q \sqrt{a}, b, c, d q^{k}, a q^{-k} / c, a q^{1+N} / b, a q^{-N} / d \\
\sqrt{a},-\sqrt{a}, a q / b, a q / c, a q^{1-k} / d, c q^{1+k}, b q^{-N}, d q^{1+N} ; q, q
\end{array}\right]
$$




$$
\begin{gathered}
=\frac{(a q / b c, c q / b, d q, d q / a ; q)_{N}}{(c d q / a, d q / c, q / b, a q / b ; q)_{N}} \frac{\left(c d / a, b d / a, c q, c q / a, d q^{1+N} / b, q^{-N} ; q\right)_{k}}{\left(q, c q / b, d / a, d, b c q^{-N} / a, c d q^{1+N} / a ; q\right)_{k}} \\
\times \frac{(q, q, a q, q / a, c d q / a, a q / c d, c q / d, d q / c ; q)_{\infty}}{(c q, q / c, d q, q / d, c q / a, a q / c, d q / a, a q / d ; q)_{\infty}}
\end{gathered}
$$

Note that two of the upper parameters of the ${ }_{8} \psi_{8}$ series in (4.3) differ multiplicatively from corresponding lower parameters by $q^{N}$, a nonnegative integral power of $q$.

Although, to the best of our knowledge, Theorem 4.1 is stated here explicitly for the first time, there is also another way to derive (or verify) (4.3) which we just sketch very briefly. By adequately specializing M. Jackson's [25, Eq. (2.2)] transformation formula for a very-well-poised ${ }_{8} \psi_{8}$ series into a sum of two ${ }_{8} \phi_{7}$ series (cf. [19, Eq. (5.6.2)]), one of the right hand side terms (a multiple of an ${ }_{8} \phi_{7}$ series) becomes zero, while the other ${ }_{8} \phi_{7}$ series can be summed by an application of F. H. Jackson's terminating ${ }_{8} \phi_{7}$ summation. We omit displaying the details.

Our second application involves the nonterminating very-well-poised ${ }_{8} \phi_{7}$ summation in (2.4) which, by introducing an additional integer $l$, can be written as

$$
\begin{aligned}
& { }_{8} \phi_{7}\left[\begin{array}{l}
a, q \sqrt{a},-q \sqrt{a}, c q^{l}, a q^{-l} / b,-(a b q / c)^{1 / 2},(a b q / c)^{1 / 2}, c / b \\
\sqrt{a},-\sqrt{a}, a q^{1-l} / c, b q^{1+l},-(a c q / b)^{1 / 2},(a c q / b)^{1 / 2}, a b q / c
\end{array} ; q,-\frac{b q}{c}\right] \\
& =\frac{(a q, b q / c ; q)_{\infty}}{\left(a q^{1-l} / c, b q^{1+l} ; q\right)_{\infty}} \frac{\left(c q^{1+l}, a q^{1-l} / b, a b q^{2-l} / c^{2}, b^{2} q^{2+l} / c ; q^{2}\right)_{\infty}}{\left(q, a c q / b, a b q^{2} / c, b^{2} q^{2} / c^{2} ; q^{2}\right)_{\infty}},
\end{aligned}
$$

where $|b q / c|<1$. Note that on the right hand side we have $q$ and $q^{2}$ appearing as bases. Thus, we may refer to (4.4) as a quadratic summation. We may rewrite (4.4) as

$$
\begin{gathered}
\sum_{k \in \mathbb{Z}} \underbrace{\frac{\left(1-a q^{2 k}\right)}{(1-a)} \frac{(c ; q)_{k+l}(a / b ; q)_{k-l}}{(b q ; q)_{k+l}(a q / c ; q)_{k-l}} q^{k-l}}_{g_{k l}} \underbrace{\frac{(a, c / b ; q)_{k}}{(q, a b q / c ; q)_{k}} \frac{\left(a b q / c ; q^{2}\right)_{k}}{\left(a c q / b ; q^{2}\right)_{k}}\left(-\frac{b}{c}\right)^{k}}_{b_{k}} \\
=\underbrace{\frac{(c ; q)_{l}(a / b ; q)_{-l}(a q, b q / c ; q)_{\infty}\left(c q^{1+l}, a q^{1-l} / b, a b q^{2-l} / c^{2}, b^{2} q^{2+l} / c ; q^{2}\right)_{\infty}}{q^{l}(a q / c, b q ; q)_{\infty}\left(q, a c q / b, a b q^{2} / c, b^{2} q^{2} / c^{2} ; q^{2}\right)_{\infty}}}_{a_{l}}
\end{gathered}
$$

by which we have established (2.11) for the above choices of $a_{l}$ and $b_{k}$. By virtue of the matrix inversion in Theorem 3.1 and the equivalence of (2.10) and (2.11), we immediately deduce from (4.5) the following inverse relation:

$$
\begin{gathered}
\sum_{n \in \mathbb{Z}} \underbrace{\frac{(a q / b, b q / a, a q / c, c q / a, b q, q / b, c q, q / c ; q)_{\infty}\left(1-b c q^{2 n} / a\right)(b ; q)_{n+k}(a / c ; q)_{k-n}}{(q, q, a q, q / a, a q / b c, b c q / a, c q / b, b q / c ; q)_{\infty}(1-b c / a)(c q ; q)_{n+k}(a q / b ; q)_{k-n}}}_{f_{n k}} \\
\quad \times \underbrace{\frac{(c ; q)_{n}(a / b ; q)_{-n}(a q, b q / c ; q)_{\infty}\left(c q^{1+n}, a q^{1-n} / b, a b q^{2-n} / c^{2}, b^{2} q^{2+n} / c ; q^{2}\right)_{\infty}}{q^{n}(a q / c, b q ; q)_{\infty}\left(q, a c q / b, a b q^{2} / c, b^{2} q^{2} / c^{2} ; q^{2}\right)_{\infty}}}_{a_{n}}
\end{gathered}
$$




$$
=\underbrace{\frac{(a, c / b ; q)_{k}}{(q, a b q / c ; q)_{k}} \frac{\left(a b q / c ; q^{2}\right)_{k}}{\left(a c q / b ; q^{2}\right)_{k}}\left(-\frac{b}{c}\right)^{k}}_{b_{k}}
$$

(subject to the convergence condition $|q|<1$ ). After substitution of variables (simultaneously $a \mapsto b c / a, b \mapsto c$, and $c \mapsto b$ ), we obtain the following bilateral quadratic summation:

Theorem 4.2 Let $a, b$, and $c$ be indeterminates, and let $k$ be an arbitrary integer. Then

$$
\begin{aligned}
\left.\sum_{n=-\infty}^{\infty} \frac{\left(1-a q^{2 n}\right)}{(1-a)} \frac{(}{\left(a q, c q^{k}, a q^{-k} / b ; q\right)_{n}} q^{\left(\begin{array}{c}
n \\
2
\end{array}\right)}\left(-\frac{a q}{c}\right)^{n} / c, b q^{1+k} ; q\right)_{n} \\
\quad \times\left(b q^{1+n}, b q^{1-n} / a, c^{2} q^{2+n} / b, c^{2} q^{2-n} / a b ; q^{2}\right)_{\infty} \\
=\frac{(b c / a, b q, b q / a, b / c ; q)_{k}}{\left(q, c / a, c, c^{2} q / a ; q\right)_{k}} \frac{\left(c^{2} q / a ; q^{2}\right)_{k}}{\left(b^{2} q / a ; q^{2}\right)_{k}}\left(-\frac{c}{b}\right)^{k} \\
\times \frac{(q, q, a q, q / a, a q / b c, b q / c ; q)_{\infty}}{(b q, q / b, b q / a, a q / b, q / c, a q / c ; q)_{\infty}}\left(q, b^{2} q / a, c^{2} q^{2} / a, c^{2} q^{2} / b^{2} ; q^{2}\right)_{\infty}
\end{aligned}
$$

Note that the series in (4.6) converges absolutely for $|q|<1$. This can easily be seen by splitting the sum in two series according to the parity of the index of summation $(n=2 m$ or $n=2 m+1$ for integer $m$ ). In this case it is also easy to rewrite the whole sum as a sum of two basic hypergeometric series with base $q^{2}$, where each sum is absolutely convergent for $\left|q^{2}\right|<1$ (i.e., $|q|<1$ ).

Our third application involves Shukla's very-well-poised ${ }_{8} \psi_{8}$ summation (2.5). By introducing an additional integer $l$, we can deduce from (2.5) the identity

$$
\begin{array}{r}
{ }_{8} \psi_{8}\left[\begin{array}{c}
q \sqrt{a},-q \sqrt{a}, c q^{l}, a q^{-l} / b, d, e, u, a q^{2} / u \\
\sqrt{a},-\sqrt{a}, a q^{1-l} / c, b q^{1+l}, a q / d, a q / e, a q / u, u / q
\end{array} ;, \frac{a b}{c d e}\right] \\
=\left(1-\frac{(1-c / b)\left(1-c d q^{l} / a\right)\left(1-c e q^{l} / a\right)}{\left(1-c q^{1+l} / u\right)\left(1-c u q^{l-1} / a\right)(1-c d e / a b)}\right) \\
\times \frac{\left(1-u q^{-1-l} / c\right)\left(1-c u q^{l-1} / a\right)}{(1-u / a q)(1-u / q)}\left(\frac{a}{d e}\right)^{l} \frac{(c d / a, c e / a, b q, b q / a ; q)_{l}}{(c / a, c, b q / d, b q / e ; q)_{l}} \\
\times \frac{(q, a q, q / a, b q / c, a q / c d, a q / c e, b q / d, b q / e, a q / d e ; q)_{\infty}}{(a q / c, b q, a q / d, a q / e, q / c, b q / a, q / d, q / e, a b q / c d e ; q)_{\infty}},
\end{array}
$$

where $|a b / c d e|<1$. We may rewrite (4.7) in the form (2.11) with $g_{k l}$ as in (3.2),

$$
\begin{aligned}
a_{l}=\left(1-\frac{(1-c / b)\left(1-c d q^{l} / a\right)\left(1-c e q^{l} / a\right)}{\left(1-c q^{1+l} / u\right)\left(1-c u q^{l-1} / a\right)(1-c d e / a b)}\right) \\
\quad \times \frac{\left(1-u q^{-1-l} / c\right)\left(1-c u q^{l-1} / a\right)}{(1-u / a q)(1-u / q)}\left(\frac{a b}{c d e}\right)^{l} \frac{(c d / a, c e / a ; q)_{l}}{(b q / d, b q / e ; q)_{l}}
\end{aligned}
$$




$$
\times \frac{(q, a q, q / a, b q / c, a q / c d, a q / c e, b q / d, b q / e, a q / d e ; q)_{\infty}}{(a q / c, b q, a q / d, a q / e, q / c, b q / a, q / d, q / e, a b q / c d e ; q)_{\infty}}
$$

and

$$
b_{k}=\frac{\left(d, e, u, a q^{2} / u ; q\right)_{k}}{(a q / d, a q / e, a q / u, u / q ; q)_{k}}\left(\frac{a b}{c d e q}\right)^{k} .
$$

This implies the inverse relation (2.10) with $f_{n k}$ as in (3.1), and the above choices of $a_{n}$ and $b_{k}$. This inverse relation is equivalent to

$$
\begin{aligned}
\sum_{n=-\infty}^{\infty} \frac{\left(1-b c q^{2 n} / a\right)}{(1-b c / a)} \frac{\left(b q^{k}, b q^{-k} / a, c d q / a, c e q / a ; q\right)_{n}}{\left(c q^{1-k} / a, c q^{1+k}, b q / d, b q / e ; q\right)_{n}}\left(\frac{a}{d e}\right)^{n} \\
\times\left(1-\frac{(1-c d e / a b)\left(1-c q^{n+1} / u\right)\left(1-c u q^{n-1} / a\right)}{(1-c / b)\left(1-c d q^{n} / a\right)\left(1-c e q^{n} / a\right)}\right) \\
=\frac{\left(1-u q^{k-1}\right)\left(1-a q^{k+1} / u\right)}{(b-c)}\left(\frac{a b}{c d e q}\right)^{k} \frac{(c q, a q / b, d, e ; q)_{k}}{(a / c, b, a q / d, a q / e ; q)_{k}} \\
\quad \times \frac{(q, a q / d, a q / e, q / d, q / e, c q / b, a q / b c, b c q / a, a b / c d e ; q)_{\infty}}{(a / c d, a / c e, b q / d, b q / e, q / b, a q / b, c q, c q / a, a q / d e ; q)_{\infty}}
\end{aligned}
$$

provided $|a / d e|<1$. Now, it is not difficult to see that the integer parameter $k$ in (4.8) can be eliminated. Hence, we could readily set $k=0$. However, our following substitutions not only serve to eliminate the superfluous $k$ but also serve to obtain more symmetry of the parameters. Specifically, in (4.8) we perform the simultaneous substitutions $a \mapsto b q^{-2 k} / c$, $b \mapsto b q^{-k}, c \mapsto a q^{-k} / c, d \mapsto b d q^{-k} / a, e \mapsto b e q^{-k} / a, u \mapsto a q^{1-k} / c u$, simplify further and obtain:

Theorem 4.3 Let $a, b, c, d, e$, and $u$ be indeterminates. Then

$$
\begin{aligned}
& \sum_{n=-\infty}^{\infty} \frac{\left(1-a q^{2 n}\right)}{(1-a)} \frac{(b, c ; q)_{n}}{(a q / b, a q / c ; q)_{n}} \frac{(d q, e q ; q)_{n}}{(a q / d, a q / e ; q)_{n}}\left(\frac{a^{2}}{b c d e}\right)^{n} \\
& \quad \times\left(1-\frac{(1-d e / a)\left(1-u q^{n}\right)\left(1-a^{2} q^{n} / b c u\right)}{(1-a / b c)\left(1-d q^{n}\right)\left(1-e q^{n}\right)}\right) \\
& =\frac{(1-a / c u)(1-b u / a)(q, a q, q / a, a q / b c, a q / b d, a q / b e, a q / c d, a q / c e, a / d e ; q)_{\infty}}{(b-a / c)\left(q / b, a q / b, q / c, a q / c, 1 / d, a q / d, 1 / e, a q / e, a^{2} q / b c d e ; q\right)_{\infty}}
\end{aligned}
$$

provided $\left|a^{2} / b c d e\right|<1$.

Note that Theorem 4.3 reduces to Bailey's very-well-poised ${ }_{6} \psi_{6}$ summation (1.1) if we let $u \rightarrow e$, and then perform the substitution $e \mapsto e / q$.

Remark 4.4 Instead of inverting Shukla's very-well-poised ${ }_{8} \psi_{8}$ summation (2.5), we could also have inverted a more general identity to obtain an even more general result. 
A good candidate is the $k=2$ special case of a ${ }_{2 k+4} \psi_{2 k+4}$ transformation formula due to Milne [29, Theorem 1.7] which can be written as follows:

$$
\begin{gathered}
{ }_{8} \psi_{8}\left[\begin{array}{c}
q \sqrt{a},-q \sqrt{a}, b, c, d, e, f, a q^{1+N} / f \\
\left.\sqrt{a},-\sqrt{a}, a q / b, a q / c, a q / d, a q / e, a q / f, f q^{-N} ; q, \frac{a^{2} q^{1-N}}{b c d e}\right] \\
=\frac{(q, a q, q / a, a q / b c, a q / b d, a q / b e, a q / c d, a q / c e, a q / d e ; q)_{\infty}}{\left(a q / b, a q / c, a q / d, a q / e, q / b, q / c, q / d, q / e, a^{2} q / b c d e ; q\right)_{\infty}} \\
\times \frac{(b q / f, a q / b f ; q)_{N}}{(a q / f, q / f ; q)_{N}}{ }_{4} \phi_{3}\left[\begin{array}{c}
q^{-N}, b c / a, b d / a, b e / a \\
b q / f, b f q^{-N} / a, b c d e / a^{2}
\end{array} ; q, q\right]
\end{array},\right.
\end{gathered}
$$

where $\left|a^{2} q^{1-N} / b c d e\right|<1$, for convergence. Note that for $N=0$ (4.10) reduces to (1.1), whereas (2.5) is just the special case $N=1$ of (4.10) where the balanced ${ }_{4} \phi_{3}$ series on the right hand side reduces to a sum of two terms only. Another noteworthy special case is obtained by letting $e \rightarrow a / b$ in (4.10). In this case, the ${ }_{4} \phi_{3}$ series on the right hand side is just one and the above transformation reduces to a summation formula for an ${ }_{8} \psi_{8}$ series (which in an equivalent form is displayed in (5.8)). A similar specialization in the full Theorem 1.7 of Milne [29], without the restriction $k=2$, yields a summation for a $q$-IPD type (or $q$-Karlsson-Minton type; "IPD" stands for integral parameter differences, cf. [37],[39, Sec. 7]) very-well-poised ${ }_{2 k+4} \psi_{2 k+4}$ series, a summation apparently originally missed by Milne, but later independently (re-)discovered by Chu [16, Theorem 12].

However, it is clear that it is always possible to obtain more general results. The reason that in our last application of this section we only inverted (2.5) and not the more general (4.10) is that we feel that Theorem 4.3, which already seems to be new, looks appealing enough, in spite, or because, of the relative simplicity of the explicitly given terms. We leave it as an easy exercise for the reader to carry out the analysis of inverting (4.10) to obtain a result which is more general than Theorem 4.3.

\section{$5 \quad$ More identities}

We can use the newly derived summations in Section 4 to derive other identities. The machinery we apply is very elementary. In our summations involving an integer parameter $k$, we multiply both sides by a suitable expression depending on $k$ and then sum both sides of the identity over all integers $k$. We will then have a double sum on one side of the identity. There we interchange summations and simplify the inner sum. As result we obtain a transformation of series.

It should be understood that the identities we are deriving by the described method (by combining various summations) are definitely not an exhaustive list of all possibilities. We just give a number of samples which seem to be interesting enough to present. 


\subsection{Some consequences of Theorem 4.1}

First, we combine Theorem 4.1 with itself. We multiply both sides of (4.3) by

$$
\frac{\left(1-c d q^{2 k} / a\right)}{(1-c d / a)} \frac{\left(d / a, d, e, c, c d q^{1+M} / a e, c q^{-M} / a ; q\right)_{k}}{\left(c q, c q / a, c d q / a e, d q / a, e q^{-M}, d q^{1+M} ; q\right)_{k}} q^{k}
$$

and sum over all integers $k$. On the right hand side we obtain

$$
\begin{aligned}
& \frac{(a q / b c, c q / b, d q, d q / a ; q)_{N}}{(c d q / a, d q / c, q / b, a q / b ; q)_{N}} \frac{(q, q, a q, q / a, c d q / a, a q / c d, c q / d, d q / c ; q)_{\infty}}{(c q, q / c, d q, q / d, c q / a, a q / c, d q / a, a q / d ; q)_{\infty}} \\
& \times{ }_{10} \phi_{9}\left[\begin{array}{l}
c d / a, q(c d / a)^{\frac{1}{2}},-q(c d / a)^{\frac{1}{2}}, b d / a, c, e, \\
(c d / a)^{\frac{1}{2}},-(c d / a)^{\frac{1}{2}}, c q / b, d q / a, c d q / a e,
\end{array}\right. \\
& \left.\begin{array}{c}
c d q^{1+M} / a e, c q^{-M} / a, d q^{1+N} / b, q^{-N} \\
e q^{-M}, d q^{1+M}, b c q^{-N} / a, c d q^{1+N} / a
\end{array} ; q, q\right] .
\end{aligned}
$$

On the left hand side we obtain

$$
\begin{aligned}
\sum_{k=-\infty}^{\infty} \frac{\left(1-c d q^{2 k} / a\right)}{(1-c d / a)} \frac{\left(d / a, d, e, c, c d q^{1+M} / a e, c q^{-M} / a ; q\right)_{k}}{\left(c q, c q / a, c d q / a e, d q / a, e q^{-M}, d q^{1+M} ; q\right)_{k}} q^{k} \\
\times \sum_{j=-\infty}^{\infty} \frac{\left(1-a q^{2 j}\right)}{(1-a)} \frac{\left(b, c, d q^{k}, a q^{-k} / c, a q^{1+N} / b, a q^{-N} / d ; q\right)_{j}}{\left(a q / b, a q / c, a q^{1-k} / d, c q^{1+k}, b q^{-N}, d q^{1+N} ; q\right)_{j}} q^{j} \\
=\sum_{j=-\infty}^{\infty} \frac{\left(1-a q^{2 j}\right)}{(1-a)} \frac{\left(b, c, d, a / c, a q^{1+N} / b, a q^{-N} / d ; q\right)_{j}}{\left(a q / b, a q / c, a q / d, c q, b q^{-N}, d q^{1+N} ; q\right)_{j}} q^{j} \\
\times \sum_{k=-\infty}^{\infty} \frac{\left(1-c d q^{2 k} / a\right)}{(1-c d / a)} \frac{\left(d q^{-j} / a, d q^{j}, e, c, c d q^{1+M} / a e, c q^{-M} / a ; q\right)_{k}}{\left(c q^{1+j}, c q^{1-j} / a, c d q / a e, d q / a, e q^{-M}, d q^{1+M} ; q\right)_{k}} q^{k}
\end{aligned}
$$

Now the inner sum can be evaluated by (4.3) and we obtain

$$
\begin{gathered}
\sum_{j=-\infty}^{\infty} \frac{\left(1-a q^{2 j}\right)}{(1-a)} \frac{\left(b, c, d, a / c, a q^{1+N} / b, a q^{-N} / d ; q\right)_{j}}{\left(a q / b, a q / c, a q / d, c q, b q^{-N}, d q^{1+N} ; q\right)_{j}} q^{j} \\
\quad \times \frac{(d q / a e, c q / e, d q, a q / c ; q)_{M}}{(a q, d q / c, q / e, c d q / a e ; q)_{M}} \frac{\left(a, a e / c, c q, a q / d, d q^{1+M} / e, q^{-M} ; q\right)_{j}}{\left(q, c q / e, a / c, d, a e q^{-M} / d, a q^{1+M} ; q\right)_{j}} \\
\quad \times \frac{(q, q, c d q / a, a q / c d, a q, q / a, c q / d, d q / c ; q)_{\infty}}{(c q, q / c, d q, q / d, a q / d, d q / a, a q / c, c q / a ; q)_{\infty}} \\
=\frac{(q, q, c d q / a, a q / c d, a q, q / a, c q / d, d q / c ; q)_{\infty}}{(c q, q / c, d q, q / d, a q / d, d q / a, a q / c, c q / a ; q)_{\infty}} \frac{(d q, e q, d q, a q / c ; q)_{M}}{(a q, d q / e, c d q / a e ; q)_{M}} \\
\times{ }_{10} \phi_{9}\left[\begin{array}{l}
a, q a^{\frac{1}{2}},-q a^{\frac{1}{2}}, b, c, a e / c, a q^{1+N} / b, a q^{-N} / d, d q^{1+M} / e, q^{-M} \\
a^{\frac{1}{2}},-a^{\frac{1}{2}}, a q / b, a q / c, c q / e, b q^{-N}, d q^{1+N}, a e q^{-M} / d, a q^{1+M}
\end{array} q, q\right]
\end{gathered}
$$

Equating the last expression with (5.1), and performing the simultaneous substitutions $d \mapsto e$ and $e \mapsto c d / a$, we obtain the following terminating very-well-poised balanced ${ }_{10} \phi_{9}$ transformation: 
Corollary 5.1 Let $a, b, c, d$, and e, be indeterminates, and let $N$ and $M$ be nonnegative integers. Then

$$
\begin{gathered}
{ }_{10} \phi_{9}\left[\begin{array}{c}
a, q a^{\frac{1}{2}},-q a^{\frac{1}{2}}, b, c, d, a q^{1+N} / b, a q^{-N} / e, a e q^{1+M} / c d, q^{-M} \\
\left.a^{\frac{1}{2}},-a^{\frac{1}{2}}, a q / b, a q / c, a q / d, b q^{-N}, e q^{1+N}, c d q^{-M} / e, a q^{1+M} ; q, q\right]
\end{array}\right. \\
=\frac{(a q, e q / c, a q / c d, e q / d ; q)_{M}}{(e q / c d, a q / d, e q, a q / c ; q)_{M}} \frac{(a q / b c, c q / b, e q, e q / a ; q)_{N}}{(c e q / a, e q / c, q / b, a q / b ; q)_{N}} \\
\times{ }_{10} \phi_{9}\left[\begin{array}{c}
c e / a, q(c e / a)^{\frac{1}{2}},-q(c e / a)^{\frac{1}{2}}, b e / a, c, c d / a \\
(c e / a)^{\frac{1}{2}},-(c e / a)^{\frac{1}{2}}, c q / b, e q / a, e q / d, \\
e q^{1+M} / d, c q^{-M} / a, e q^{1+N} / b, q^{-N} \\
c d q^{-M} / a, e q^{1+M}, b c q^{-N} / a, c e q^{1+N} / a
\end{array} ; q, q\right] .
\end{gathered}
$$

It appears that Corollary 5.1 does not immediately follow by specializing either of Bailey's [11] four-term nonterminating ${ }_{10} \phi_{9}$ transformations (cf. [19, Eq. (2.12.9) and Ex. 2.30]).

Now let us combine Theorem 4.1 with the terminating Jackson summation in (2.3). We multiply both sides of (4.3) by

$$
\frac{\left(1-c d q^{2 k} / a\right)}{(1-c d / a)} \frac{\left(c d / a, d / a, d, e, c^{2} q^{1+M} / a e, q^{-M} ; q\right)_{k}}{\left(q, c q, c q / a, c d q / a e, d e q^{-M} / c, c d q^{1+M} / a ; q\right)_{k}} q^{k}
$$

and sum over all integers $k$. On the right hand side we obtain

$$
\begin{gathered}
\frac{(a q / b c, c q / b, d q, d q / a ; q)_{N}}{(c d q / a, d q / c, q / b, a q / b ; q)_{N}} \frac{(q, q, a q, q / a, c d q / a, a q / c d, c q / d, d q / c ; q)_{\infty}}{(c q, q / c, d q, q / d, c q / a, a q / c, d q / a, a q / d ; q)_{\infty}} \\
\times{ }_{10} \phi_{9}\left[\begin{array}{c}
c d / a, q(c d / a)^{\frac{1}{2}},-q(c d / a)^{\frac{1}{2}}, c d / a, b d / a, e \\
(c d / a)^{\frac{1}{2}},-(c d / a)^{\frac{1}{2}}, q, c q / b, c d q / a e \\
d q^{1+N} / b, c^{2} q^{1+M} / a e, q^{-N}, q^{-M} \\
b c q^{-N} / a, d e q^{-M} / c, c d q^{1+N} / a, c d q^{1+M} / a
\end{array} ; q, q\right] .
\end{gathered}
$$

On the left hand side we obtain

$$
\begin{gathered}
\sum_{k=-\infty}^{\infty} \frac{\left(1-c d q^{2 k} / a\right)}{(1-c d / a)} \frac{\left(c d / a, d / a, d, e, c^{2} q^{1+M} / a e, q^{-M} ; q\right)_{k}}{\left(q, c q, c q / a, c d q / a e, d e q^{-M} / c, c d q^{1+M} / a ; q\right)_{k}} q^{k} \\
\times \sum_{j=-\infty}^{\infty} \frac{\left(1-a q^{2 j}\right)}{(1-a)} \frac{\left(b, c, d q^{k}, a q^{-k} / c, a q^{1+N} / b, a q^{-N} / d ; q\right)_{j}}{\left(a q / b, a q / c, a q^{1-k} / d, c q^{1+k}, b q^{-N}, d q^{1+N} ; q\right)_{j}} q^{j} \\
=\sum_{j=-\infty}^{\infty} \frac{\left(1-a q^{2 j}\right)}{(1-a)} \frac{\left(b, c, d, a / c, a q^{1+N} / b, a q^{-N} / d ; q\right)_{j}}{\left(a q / b, a q / c, a q / d, c q, b q^{-N}, d q^{1+N} ; q\right)_{j}} q^{j} \\
\times \sum_{k=-\infty}^{\infty} \frac{\left(1-c d q^{2 k} / a\right)}{(1-c d / a)} \frac{\left(c d / a, d q^{-j} / a, d q^{j}, e, c^{2} q^{1+M} / a e, q^{-M} ; q\right)_{k}}{\left(q, c q^{1+j}, c q^{1-j} / a, c d q / a e, d e q^{-M} / c, c d q^{1+M} / a ; q\right)_{k}} q^{k}
\end{gathered}
$$


Now the inner sum can be evaluated by Jackson's very-well-poised ${ }_{8} \phi_{7}$ summation (2.3) and we obtain

$$
\begin{gathered}
\sum_{j=-\infty}^{\infty} \frac{\left(1-a q^{2 j}\right)}{(1-a)} \frac{\left(b, c, d, a / c, a q^{1+N} / b, a q^{-N} / d ; q\right)_{j}}{\left(a q / b, a q / c, a q / d, c q, b q^{-N}, d q^{1+N} ; q\right)_{j}} q^{j} \\
\times \frac{\left(c d q / a, c q / d, c q^{1+j} / e, c q^{1-j} / a e ; q\right)_{M}}{\left(c q^{1+j}, c q^{1-j} / a, c d q / a e, c q / d e ; q\right)_{M}} \\
=\frac{(c d q / a, c q / d, c q / e, c q / a e ; q)_{M}}{(c q, c q / a, c d q / a e, c q / d e ; q)_{M}} \\
\times{ }_{10} \psi_{10}\left[\begin{array}{c}
q a^{\frac{1}{2}},-q a^{\frac{1}{2}}, b, c, d, a e / c, a q^{1+N} / b, a q^{-N} / d, c q^{1+M} / e, a q^{-M} / c \\
a^{\frac{1}{2}},-a^{\frac{1}{2}}, a q / b, a q / c, a q / d, c q / e, b q^{-N}, d q^{1+N}, a e q^{-M} / c, c q^{1+M}
\end{array} q, q\right]
\end{gathered}
$$

Equating the last expression with (5.3), and performing the substitution $e \mapsto c e / a$, we obtain the following transformation for a particular very-well-poised balanced ${ }_{10} \psi_{10}$ series:

Corollary 5.2 Let $a, b, c, d$, and e, be indeterminates, and let $N$ and $M$ be nonnegative integers. Then

$$
\begin{gathered}
{ }_{10} \psi_{10}\left[\begin{array}{c}
q a^{\frac{1}{2}},-q a^{\frac{1}{2}}, b, c, d, e, a q^{1+N} / b, a q^{-N} / d, a q^{1+M} / e, a q^{-M} / c \\
\left.a^{\frac{1}{2}},-a^{\frac{1}{2}}, a q / b, a q / c, a q / d, a q / e, b q^{-N}, d q^{1+N}, e q^{-M}, c q^{1+M} ; q, q\right]
\end{array}\right] \\
=\frac{(c q, c q / a, d q / e, a q / d e ; q)_{M}}{(c d q / a, c q / d, a q / e, q / e ; q)_{M}} \frac{(a q / b c, c q / b, d q, d q / a ; q)_{N}}{(c d q / a, d q / c, q / b, a q / b ; q)_{N}} \\
\quad \times \frac{(q, q, a q, q / a, c d q / a, a q / c d, c q / d, d q / c ; q)_{\infty}}{(c q, q / c, d q, q / d, c q / a, a q / c, d q / a, a q / d ; q)_{\infty}} \\
\times{ }_{10} \phi_{9}\left[\begin{array}{c}
c d / a, q(c d / a)^{\frac{1}{2}},-q(c d / a)^{\frac{1}{2}}, c d / a, b d / a, c e / a \\
(c d / a)^{\frac{1}{2}},-(c d / a)^{\frac{1}{2}}, q, c q / b, d q / e, \\
d q^{1+M} / b, c q^{1+M} / e, q^{-N}, q^{-M} \\
b c q^{-N} / a, d e q^{-M} / a, c d q^{1+N} / a, c d q^{1+M} / a
\end{array} ; q, q\right] .
\end{gathered}
$$

Let us now combine Theorem 4.1 with Shukla's very-well-poised ${ }_{8} \psi_{8}$ summation in (2.5). We multiply both sides of (4.3) by

$$
\frac{\left(1-c d q^{2 k} / a\right)}{(1-c d / a)} \frac{(d / a, d, e, f, g q, c d q / a g ; q)_{k}}{(c q, c q / a, c d q / a e, c d q / a f, c d / a g, g ; q)_{k}}\left(\frac{c^{2}}{a e f}\right)^{k}
$$

and sum over all integers $k$. On the right hand side we obtain

$$
\begin{gathered}
\frac{(a q / b c, c q / b, d q, d q / a ; q)_{N}}{(c d q / a, d q / c, q / b, a q / b ; q)_{N}} \frac{(q, q, a q, q / a, c d q / a, a q / c d, c q / d, d q / c ; q)_{\infty}}{(c q, q / c, d q, q / d, c q / a, a q / c, d q / a, a q / d ; q)_{\infty}} \\
\times{ }_{10} \phi_{9}\left[\begin{array}{c}
c d / a, q(c d / a)^{\frac{1}{2}},-q(c d / a)^{\frac{1}{2}}, b d / a, e, f \\
(c d / a)^{\frac{1}{2}},-(c d / a)^{\frac{1}{2}}, c q / b, c d q / a e, c d q / a f \\
g q, c d q / a g, d q^{1+N} / b, q^{-N} \\
c d / a g, g, b c q^{-N} / a, c d q^{1+N} / a
\end{array} ; q, \frac{c^{2}}{a e f}\right] .
\end{gathered}
$$


On the left hand side we obtain

$$
\begin{gathered}
\sum_{k=-\infty}^{\infty} \frac{\left(1-c d q^{2 k} / a\right)}{(1-c d / a)} \frac{(d / a, d, e, f, g q, c d q / a g ; q)_{k}}{(c q, c q / a, c d q / a e, c d q / a f, c d / a g, g ; q)_{k}}\left(\frac{c^{2}}{a e f}\right)^{k} \\
\times \sum_{j=-\infty}^{\infty} \frac{\left(1-a q^{2 j}\right)}{(1-a)} \frac{\left(b, c, d q^{k}, a q^{-k} / c, a q^{1+N} / b, a q^{-N} / d ; q\right)_{j}}{\left(a q / b, a q / c, a q^{1-k} / d, c q^{1+k}, b q^{-N}, d q^{1+N} ; q\right)_{j}} q^{j} \\
=\sum_{j=-\infty}^{\infty} \frac{\left(1-a q^{2 j}\right)}{(1-a)} \frac{\left(b, c, d, a / c, a q^{1+N} / b, a q^{-N} / d ; q\right)_{j}}{\left(a q / b, a q / c, a q / d, c q, b q^{-N}, d q^{1+N} ; q\right)_{j}} q^{j} \\
\times \sum_{k=-\infty}^{\infty} \frac{\left(1-c d q^{2 k} / a\right)}{(1-c d / a)} \frac{\left(d q^{-j} / a, d q^{j}, e, f, g q, c d q / a g ; q\right)_{k}}{\left(c q^{1+j}, c q^{1-j} / a, c d q / a e, c d q / a f, c d / a g, g ; q\right)_{k}}\left(\frac{c^{2}}{a e f}\right)^{k}
\end{gathered}
$$

Now the inner sum, provided $\mid c^{2} /$ ae $f \mid<1$, can be evaluated by Shukla's very-well-poised ${ }_{8} \psi_{8}$ summation $(2.5)$ and we obtain

$$
\begin{aligned}
& \sum_{j=-\infty}^{\infty} \frac{\left(1-a q^{2 j}\right)}{(1-a)} \frac{\left(b, c, d, a / c, a q^{1+N} / b, a q^{-N} / d ; q\right)_{j}}{\left(a q / b, a q / c, a q / d, c q, b q^{-N}, d q^{1+N} ; q\right)_{j}} q^{j} \\
& \times\left(1-\frac{(1-d / c)\left(1-e q^{-j} / c\right)\left(1-f q^{-j} / c\right)}{\left(1-d q^{-j} / a g\right)\left(1-g q^{-j} / c\right)\left(1-a e f / c^{2}\right)}\right) \frac{\left(1-a g q^{j} / d\right)\left(1-g q^{-j} / c\right)}{(1-a g / c d)(1-g)} \\
& \times \frac{\left(q, c d q / a, a q / c d, c q / d, c q^{1+j} / e, c q^{1+j} / f, c q^{1-j} / a e, c q^{1-j} / a f, c d q / a e f ; q\right)_{\infty}}{\left(c q^{1+j}, c q^{1-j} / a, c d q / a e, c d q / a f, a q^{1+j} / d, q^{1-j} / d, q / e, q / f, c^{2} q / a e f ; q\right)_{\infty}} \\
&= \frac{(q, c d q / a, a q / c d, c q / d, c q / e, c q / f, c q / a e, c q / a f, c d q / a e f ; q)_{\infty}}{\left(c q, c q / a, c d q / a e, c d q / a f, a q / d, q / d, q / e, q / f, c^{2} q / a e f ; q\right)_{\infty}} \\
& \quad \times \sum_{j=-\infty}^{\infty} \frac{\left(1-a q^{2 j}\right)}{(1-a)} \frac{\left(b, c, a e / c, a f / c, a q^{1+N} / b, a q^{-N} / d ; q\right)_{j}}{\left(a q / b, a q / c, c q / e, c q / f, b q^{-N}, d q^{1+N} ; q\right)_{j}}\left(\frac{c d q}{a e f}\right)^{j} \\
& \quad \times\left(1-\frac{(1-d / c)\left(1-e q^{-j} / c\right)\left(1-f q^{-j} / c\right)}{\left(1-d q^{-j} / a g\right)\left(1-g q^{-j} / c\right)\left(1-a e f / c^{2}\right)}\right) \frac{\left(1-a g q^{j} / d\right)\left(1-g q^{-j} / c\right)}{(1-a g / c d)(1-g)} .
\end{aligned}
$$

Equating the last expression with (5.5), moving the infinite products, and performing the simultaneous substitutions $d \mapsto f, e \mapsto c d / a$, and $f \mapsto c e / a$, we obtain

Corollary 5.3 Let $a, b, c, d, e, f$, and $g$ be indeterminates, and let $N$ be a nonnegative integer. Then

$$
\begin{gathered}
\sum_{j=-\infty}^{\infty} \frac{\left(1-a q^{2 j}\right)}{(1-a)} \frac{\left(b, c, d, e, a q^{1+N} / b, a q^{-N} / f ; q\right)_{j}}{\left(a q / b, a q / c, a q / d, a q / e, b q^{-N}, f q^{1+N} ; q\right)_{j}}\left(\frac{a f q}{c d e}\right)^{j} \\
\times\left(1-\frac{(1-f / c)\left(1-d q^{-j} / a\right)\left(1-e q^{-j} / a\right)}{\left(1-f q^{-j} / a g\right)\left(1-g q^{-j} / c\right)(1-d e / a)}\right) \frac{\left(1-a g q^{j} / f\right)\left(1-g q^{-j} / c\right)}{(1-a g / c f)(1-g)} \\
\quad=\frac{(q, a q, q / a, a q / c d, a q / c e, a q / d e, f q / c, f q / d, f q / e ; q)_{\infty}}{(a q / c, a q / d, a q / e, q / c, q / d, q / e, f q, f q / a, a f q / c d e ; q)_{\infty}}
\end{gathered}
$$




$$
\begin{aligned}
& \times \frac{(a q / b c, c q / b, f q, f q / a ; q)_{N}}{(c f q / a, f q / c, q / b, a q / b ; q)_{N}} 1_{0} \phi_{9}\left[\begin{array}{c}
c f / a, q(c f / a)^{\frac{1}{2}},-q(c f / a)^{\frac{1}{2}}, \\
(c f / a)^{\frac{1}{2}},-(c f / a)^{\frac{1}{2}},
\end{array}\right.
\end{aligned}
$$

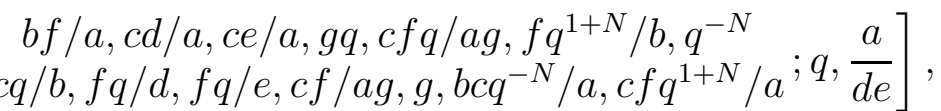

provided $\mid$ af $/$ cde $\mid<1$.

Note that we have applied analytic continuation to obtain the convergence condition in Corollary 5.3.

If we let $g \rightarrow 0$ or $g \rightarrow \infty$ in (5.6), we obtain

$$
\begin{gathered}
{ }_{8} \psi_{8}\left[\begin{array}{c}
q \sqrt{a},-q \sqrt{a}, b, c, d, e, a q^{1+N} / b, a q^{-N} / f \\
\left.\sqrt{a},-\sqrt{a}, a q / b, a q / c, a q / d, a q / e, b q^{-N}, f q^{1+N} ; q, \frac{a f q}{c d e}\right]
\end{array}\right. \\
=\frac{(q, a q, q / a, a q / c d, a q / c e, a q / d e, f q / c, f q / d, f q / e ; q)_{\infty}}{(a q / c, a q / d, a q / e, q / c, q / d, q / e, f q, f q / a, a f q / c d e ; q)_{\infty}} \\
\times \frac{(a q / b c, c q / b, f q, f q / a ; q)_{N}}{(c f q / a, f q / c, q / b, a q / b ; q)_{N}} \\
\times{ }_{8} \phi_{7}\left[\begin{array}{c}
c f / a, q(c f / a)^{\frac{1}{2}},-q(c f / a)^{\frac{1}{2}}, b f / a, c d / a, c e / a, f q^{1+N} / b, q^{-N} \\
(c f / a)^{\frac{1}{2}},-(c f / a)^{\frac{1}{2}}, c q / b, f q / d, f q / e, b c q^{-N} / a, c f q^{1+N} / a
\end{array} ; q, \frac{a q}{d e}\right]
\end{gathered}
$$

provided $|a f q / c d e|<1$. Note that two of the upper parameters in the ${ }_{8} \psi_{8}$ on the left hand side of (5.7) differ multiplicatively from corresponding lower parameters by $q^{N}$, a nonnegative integral power of $q$. If we let $e \rightarrow a / d$ in (5.7), the ${ }_{8} \phi_{7}$ on the right hand side becomes balanced and can be simplified by Jackson's ${ }_{8} \phi_{7}$ summation in (2.3). The resulting summation,

$$
\begin{aligned}
{ }_{8} \psi_{8}\left[\begin{array}{c}
q \sqrt{a},-q \sqrt{a}, b, c, d, a / d, a q^{1+N} / b, a q^{-N} / f \\
\sqrt{a},
\end{array}-\sqrt{a}, a q / b, a q / c, a q / d, d q, b q^{-N}, f q^{1+N} ; q, \frac{f q}{c}\right] \\
\quad=\frac{(q, q, a q, q / a, a q / c d, d q / c, f q / d, d f q / a ; q)_{\infty}}{(d q, q / d, a q / d, d q / a, a q / c, q / c, f q, f q / a ; q)_{\infty}} \frac{(a q / b d, d q / b, f q, f q / a ; q)_{N}}{(d f q / a, f q / d, q / b, a q / b ; q)_{N}}
\end{aligned}
$$

is originally due to H. S. Shukla [40, p. 266, final remark]. Subsequently, various farreaching extensions of (5.8) have been found, see Milne [29, Theorem 1.7], Chu [16, Theorem 12], Schlosser [39, Sec. 8],[37, Sec. 4], and Rosengren [33],[34].

\subsection{Some consequences of Theorem 4.2}

First, we combine Theorem 4.2 with Jackson's very-well-poised ${ }_{8} \phi_{7}$ summation in (2.3). We multiply both sides of (4.6) by

$$
\frac{\left(1-b c q^{2 k} / a\right)}{(1-b c / a)} \frac{\left(b c / a, c, c / a, d, b^{2} q^{1+N} / a d, q^{-N} ; q\right)_{k}}{\left(q, b q / a, b q, b c q / a d, c d q^{-N} / b, b c q^{1+N} / a ; q\right)_{k}} q^{k}
$$

and sum over all integers $k$. On the right hand side we obtain 


$$
\begin{gathered}
\frac{(q, q, a q, q / a, a q / b c, b q / c ; q)_{\infty}}{(b q, q / b, b q / a, a q / b, q / c, a q / c ; q)_{\infty}}\left(q, b^{2} q / a, c^{2} q^{2} / a, c^{2} q^{2} / b^{2} ; q^{2}\right)_{\infty} \\
\times \sum_{k=-\infty}^{\infty} \frac{\left(1-b c q^{2 k} / a\right)}{(1-b c / a)} \frac{\left(b c / a, b c / a, b / c, d, b^{2} q^{1+N} / a d, q^{-N} ; q\right)_{k}}{\left(q, q, c^{2} q / a, b c q / a d, c d q^{-N} / b, b c q^{1+N} / a ; q\right)_{k}} \\
\times \frac{\left(c^{2} q / a ; q^{2}\right)_{k}}{\left(b^{2} q / a ; q^{2}\right)_{k}}\left(-\frac{c q}{b}\right)^{k}
\end{gathered}
$$

On the left hand side we obtain

$$
\begin{aligned}
& \sum_{k=-\infty}^{\infty} \frac{\left(1-b c q^{2 k} / a\right)}{(1-b c / a)} \frac{\left(b c / a, c, c / a, d, b^{2} q^{1+N} / a d, q^{-N} ; q\right)_{k}}{\left(q, b q / a, b q, b c q / a d, c d q^{-N} / b, b c q^{1+N} / a ; q\right)_{k}} q^{k} \\
& \times \sum_{n=-\infty}^{\infty} \frac{\left(1-a q^{2 n}\right)}{(1-a)} \frac{\left(b, c q^{k}, a q^{-k} / b ; q\right)_{n}}{\left(a q / b, a q^{1-k} / c, b q^{1+k} ; q\right)_{n}} q^{\left(\begin{array}{c}
n \\
2
\end{array}\right)}\left(-\frac{a q}{c}\right)^{n} \\
& \\
& \quad \times\left(b q^{1+n}, b q^{1-n} / a, c^{2} q^{2+n} / b, c^{2} q^{2-n} / a b ; q^{2}\right)_{\infty} \\
&=\sum_{n=-\infty}^{\infty} \frac{\left(1-a q^{2 n}\right)}{(1-a)} \frac{(b, c, a / b ; q)_{n}}{(a q / b, a q / c, b q ; q)_{n}} q^{\left(\begin{array}{c}
n \\
2
\end{array}\right)}\left(-\frac{a q}{c}\right)^{n} \\
& \quad \times\left(b q^{1+n}, b q^{1-n} / a, c^{2} q^{2+n} / b, c^{2} q^{2-n} / a b ; q^{2}\right)_{\infty} \\
& \times \sum_{k=-\infty}^{\infty} \frac{\left(1-b c q^{2 k} / a\right)}{(1-b c / a)} \frac{\left(b c / a, c q^{n}, c q^{-n} / a, d, b^{2} q^{1+N} / a d, q^{-N} ; q\right)_{k}}{\left(q, b q^{1-n} / a, b q^{1+n}, b c q / a d, c d q^{-N} / b, b c q^{1+N} / a ; q\right)_{k}} q^{k}
\end{aligned}
$$

Now the inner sum can be evaluated by (2.3) and we obtain

$$
\begin{aligned}
& \sum_{n=-\infty}^{\infty} \frac{\left(1-a q^{2 n}\right)}{(1-a)} \frac{(b, c, a / b ; q)_{n}}{(a q / b, a q / c, b q ; q)_{n}} q^{\left(\begin{array}{c}
n \\
2
\end{array}\right)}\left(-\frac{a q}{c}\right)^{n} \\
& \quad \times\left(b q^{1+n}, b q^{1-n} / a, c^{2} q^{2+n} / b, c^{2} q^{2-n} / a b ; q^{2}\right)_{\infty} \frac{\left(b c q / a, b q / c, b q^{1-n} / a d, b q^{1+n} / d ; q\right)_{N}}{\left(b q^{1-n} / a, b q^{1+n}, b c q / a d, b q / c d ; q\right)_{N}} \\
& =\frac{(b c q / a, b q / c, b q / a d, b q / d ; q)_{N}}{(b q / a, b q, b c q / a d, b q / c d ; q)_{N}} \sum_{n=-\infty}^{\infty} \frac{\left(1-a q^{2 n}\right)}{(1-a)} \frac{\left(b, c, a d / b, b q^{1+N} / d ; q\right)_{n}}{\left(a q / b, a q / c, b q / d, a d q^{-N} / b ; q\right)_{n}} \\
& \quad \times \frac{\left(a q^{-N} / b ; q\right)_{n}}{\left(b q^{1+N} ; q\right)_{n}} q^{\left(\begin{array}{c}
n \\
2
\end{array}\right)}\left(-\frac{a q}{c}\right)^{n}\left(b q^{1+n}, b q^{1-n} / a, c^{2} q^{2+n} / b, c^{2} q^{2-n} / a b ; q^{2}\right)_{\infty}
\end{aligned}
$$

Equating the last expression with (5.9), and performing the substitution $d \mapsto b d / a$, we obtain the following bilateral quadratic transformation:

Corollary 5.4 Let $a, b, c$, and $d$ be indeterminates, and let $N$ be a nonnegative integer. Then

$$
\sum_{n=-\infty}^{\infty} \frac{\left(1-a q^{2 n}\right)}{(1-a)} \frac{\left(b, c, d, a q^{1+N} / d, a q^{-N} / b ; q\right)_{n}}{\left(a q / b, a q / c, a q / d, d q^{-N}, b q^{1+N} ; q\right)_{n}} q^{\left(\begin{array}{c}
n \\
2
\end{array}\right)}\left(-\frac{a q}{c}\right)^{n}
$$




$$
\begin{gathered}
\times\left(b q^{1+n}, b q^{1-n} / a, c^{2} q^{2+n} / b, c^{2} q^{2-n} / a b ; q^{2}\right)_{\infty} \\
=\frac{(q, q, a q, q / a, a q / b c, b q / c ; q)_{\infty}}{(b q, q / b, b q / a, a q / b, q / c, a q / c ; q)_{\infty}}\left(q, b^{2} q / a, c^{2} q^{2} / a, c^{2} q^{2} / b^{2} ; q^{2}\right)_{\infty} \\
\times \frac{(b q / a, b q, c q / d, a q / c d ; q)_{N}}{(b c q / a, b q / c, q / d, a q / d ; q)_{N}}{ }_{0} \phi_{9}\left[\begin{array}{c}
b c / a, q(b c / a)^{\frac{1}{2}},-q(b c / a)^{\frac{1}{2}} \\
(b c / a)^{\frac{1}{2}},-(b c / a)^{\frac{1}{2}}, \\
b c / a, b / c, b d / a, c(q / a)^{\frac{1}{2}},-c(q / a)^{\frac{1}{2}}, b q^{1+N} / d, q^{-N} \\
q, c^{2} q / a, c q / d, b(q / a)^{\frac{1}{2}},-b(q / a)^{\frac{1}{2}}, c d q^{-N} / a, b c q^{1+N} / a
\end{array} ;,-\frac{c q}{b}\right] .
\end{gathered}
$$

Note that the bilateral sum on the left hand side of (5.10) converges absolutely for $|q|<1$. See Theorem 4.2 for a very similar situation.

Now let us combine Theorem 4.2 with Shukla's very-well-poised ${ }_{8} \psi_{8}$ summation (2.5). We multiply both sides of (4.6) by

$$
\frac{\left(1-b c q^{2 k} / a\right)}{(1-b c / a)} \frac{(c, c / a, d, e, f q, b c q / a f ; q)_{k}}{(b q / a, b q, b c q / a d, b c q / a e, b c / a f, f ; q)_{k}}\left(\frac{b^{2}}{a d e}\right)^{k}
$$

and sum over all integers $k$. On the right hand side we obtain

$$
\begin{aligned}
& \frac{(q, q, a q, q / a, a q / b c, b q / c ; q)_{\infty}}{(b q, q / b, b q / a, a q / b, q / c, a q / c ; q)_{\infty}}\left(q, b^{2} q / a, c^{2} q^{2} / a, c^{2} q^{2} / b^{2} ; q^{2}\right)_{\infty} \\
& \times \sum_{k=-\infty}^{\infty} \frac{\left(1-b c q^{2 k} / a\right)}{(1-b c / a)} \frac{(b c / a, b / c, d, e, f q, b c q / a f ; q)_{k}}{\left(q, c^{2} q / a, b c q / a d, b c q / a e, b c / a f, f ; q\right)_{k}} \\
& \times \frac{\left(c^{2} q / a ; q^{2}\right)_{k}}{\left(b^{2} q / a ; q^{2}\right)_{k}}\left(-\frac{b c}{a d e}\right)^{k} .
\end{aligned}
$$

On the left hand side we obtain

$$
\begin{aligned}
& \sum_{k=-\infty}^{\infty} \frac{\left(1-b c q^{2 k} / a\right)}{(1-b c / a)} \frac{(c, c / a, d, e, f q, b c q / a f ; q)_{k}}{(b q / a, b q, b c q / a d, b c q / a e, b c / a f, f ; q)_{k}}\left(\frac{b^{2}}{a d e}\right)^{k} \\
& \times \sum_{n=-\infty}^{\infty} \frac{\left(1-a q^{2 n}\right)}{(1-a)} \frac{\left(b, c q^{k}, a q^{-k} / b ; q\right)_{n}}{\left(a q / b, a q^{1-k} / c, b q^{1+k} ; q\right)_{n}} q^{\left(\begin{array}{c}
n \\
2
\end{array}\right)}\left(-\frac{a q}{c}\right)^{n} \\
& \\
& \quad \times\left(b q^{1+n}, b q^{1-n} / a, c^{2} q^{2+n} / b, c^{2} q^{2-n} / a b ; q^{2}\right)_{\infty} \\
&=\sum_{n=-\infty}^{\infty} \frac{\left(1-a q^{2 n}\right)}{(1-a)} \frac{(b, c, a / b ; q)_{n}}{(a q / b, a q / c, b q ; q)_{n}} q^{\left(\begin{array}{c}
n \\
2
\end{array}\right)}\left(-\frac{a q}{c}\right)^{n} \\
& \quad \times\left(b q^{1+n}, b q^{1-n} / a, c^{2} q^{2+n} / b, c^{2} q^{2-n} / a b ; q^{2}\right)_{\infty} \\
& \times \sum_{k=-\infty}^{\infty} \frac{\left(1-b c q^{2 k} / a\right)}{(1-b c / a)} \frac{\left(c q^{n}, c q^{-n} / a, d, e, f q, b c q / a f ; q\right)_{k}}{\left(b q^{1-n} / a, b q^{1+n}, b c q / a d, b c q / a e, b c / a f, f ; q\right)_{k}}\left(\frac{b^{2}}{a d e}\right)^{k} .
\end{aligned}
$$

Now the inner sum, provided $\left|b^{2} / a d e\right|<1$, can be evaluated by (2.5) and we obtain 


$$
\begin{aligned}
& \sum_{n=-\infty}^{\infty} \frac{\left(1-a q^{2 n}\right)}{(1-a)} \frac{(b, c, a / b ; q)_{n}}{(a q / b, a q / c, b q ; q)_{n}} q^{\left(\begin{array}{c}
n \\
2
\end{array}\right)}\left(-\frac{a q}{c}\right)^{n} \\
& \times\left(b q^{1+n}, b q^{1-n} / a, c^{2} q^{2+n} / b, c^{2} q^{2-n} / a b ; q^{2}\right)_{\infty} \\
& \times\left(1-\frac{(1-c / b)\left(1-a d q^{n} / b\right)\left(1-a e q^{n} / b\right)}{\left(1-c q^{n} / f\right)\left(1-a f q^{n} / b\right)\left(1-a d e / b^{2}\right)}\right) \frac{\left(1-f q^{-n} / c\right)\left(1-a f q^{n} / b\right)}{(1-a f / b c)(1-f)} \\
& \times \frac{\left(q, b c q / a, a q / b c, b q / c, b q^{1-n} / a d, b q^{1-n} / a e, b q^{1+n} / d, b q^{1+n} / e, b c q / a d e ; q\right)_{\infty}}{\left(b q^{1-n} / a, b q^{1+n}, b c q / a d, b c q / a e, q^{1-n} / c, a q^{1+n} / c, q / d, q / e, b^{2} q / a d e ; q\right)_{\infty}} \\
& =\frac{(q, b c q / a, a q / b c, b / c, b q / a d, b q / a e, b q / d, b q / e, b c q / a d e ; q)_{\infty}}{\left(b q / a, b q, b c q / a d, b c q / a e, q / c, a q / c, q / d, q / e, b^{2} / a d e ; q\right)_{\infty}} \\
& \times \frac{b f}{a d e(1-f)(1-a f / b c)} \sum_{n=-\infty}^{\infty} \frac{\left(1-a q^{2 n}\right)}{(1-a)} \frac{(b, a d q / b, a e q / b ; q)_{n}}{(a q / b, b q / d, b q / e ; q)_{n}} q^{\left(\begin{array}{c}
n \\
2
\end{array}\right)}\left(-\frac{b}{d e}\right)^{n} \\
& \times\left(b q^{1+n}, b q^{1-n} / a, c^{2} q^{2+n} / b, c^{2} q^{2-n} / a b ; q^{2}\right)_{\infty} \\
& \times\left(1-\frac{\left(1-c q^{n} / f\right)\left(1-a f q^{n} / b\right)\left(1-a d e / b^{2}\right)}{(1-c / b)\left(1-a d q^{n} / b\right)\left(1-a e q^{n} / b\right)}\right) .
\end{aligned}
$$

Equating the last expression with (5.11), and performing the simultaneous substitutions $c \mapsto e, d \mapsto b c / a$ and $e \mapsto b d / a$, we obtain the following bilateral quadratic transformation:

Corollary 5.5 Let $a, b, c, d, e$, and $f$ be indeterminates. Then

$$
\begin{aligned}
& \sum_{n=-\infty}^{\infty} \frac{\left(1-a q^{2 n}\right)}{(1-a)} \frac{(b, c q, d q ; q)_{n}}{(a q / b, a q / c, a q / d ; q)_{n}} q^{\left(\begin{array}{c}
n \\
2
\end{array}\right)}\left(-\frac{a^{2}}{b c d}\right)^{n} \\
& \times\left(b q^{1+n}, b q^{1-n} / a, e^{2} q^{2+n} / b, e^{2} q^{2-n} / a b ; q^{2}\right)_{\infty} \\
& \times\left(1-\frac{\left(1-e q^{n} / f\right)\left(1-a f q^{n} / b\right)(1-c d / a)}{(1-e / b)\left(1-c q^{n}\right)\left(1-d q^{n}\right)}\right) \\
& =\frac{b c d(1-f)(1-a f / b e)(q, a q, q / a, e q / c, e q / d, a q / b c, a q / b d, a / c d ; q)_{\infty}}{a f(1-b / e)(q / b, a q / b, b e q / a, q / c, q / d, a q / c, a q / d, a e q / b c d ; q)_{\infty}} \\
& \times\left(q, b^{2} q / a, e^{2} q^{2} / a, e^{2} q^{2} / b^{2} ; q^{2}\right)_{\infty 10} \phi_{9}\left[\begin{array}{c}
b e / a, q(b e / a)^{\frac{1}{2}},-q(b e / a)^{\frac{1}{2}}, \\
(b e / a)^{\frac{1}{2}},-(b e / a)^{\frac{1}{2}},
\end{array}\right. \\
& \begin{array}{l}
b / e, b c / a, b d / a, f q, b e q / a f, e(q / a)^{\frac{1}{2}},-e(q / a)^{\frac{1}{2}} \\
\left.e^{2} q / a, e q / c, e q / d, b e / a f, f, b(q / a)^{\frac{1}{2}},-b(q / a)^{\frac{1}{2}} ; q,-\frac{a e}{b c d}\right],
\end{array}
\end{aligned}
$$

provided $|a e / b c d|<1$.

Note that we have applied analytic continuation to obtain the convergence condition in Corollary 5.5.

If we let multiply both sides of (5.12) by $f$ and then let $f \rightarrow 0$, we obtain

$$
\sum_{n=-\infty}^{\infty} \frac{\left(1-a q^{2 n}\right)}{(1-a)} \frac{(b, c, d ; q)_{n}}{(a q / b, a q / c, a q / d ; q)_{n}} q^{\left(\begin{array}{c}
n \\
2
\end{array}\right)}\left(-\frac{a^{2} q}{b c d}\right)^{n}
$$




$$
\begin{gathered}
\times\left(b q^{1+n}, b q^{1-n} / a, e^{2} q^{2+n} / b, e^{2} q^{2-n} / a b ; q^{2}\right)_{\infty} \\
=\frac{(q, a q, q / a, e q / c, e q / d, a q / b c, a q / b d, a q / c d ; q)_{\infty}}{(q / b, a q / b, b e q / a, q / c, q / d, a q / c, a q / d, a e q / b c d ; q)_{\infty}} \\
\times\left(q, b^{2} q / a, e^{2} q^{2} / a, e^{2} q^{2} / b^{2} ; q^{2}\right)_{\infty} \phi_{7}\left[\begin{array}{c}
b e / a, q(b e / a)^{\frac{1}{2}},-q(b e / a)^{\frac{1}{2}}, \\
(b e / a)^{\frac{1}{2}},-(b e / a)^{\frac{1}{2}},
\end{array}\right. \\
b / e, b c / a, b d / a, e(q / a)^{\frac{1}{2}},-e(q / a)^{\frac{1}{2}} \\
\left.e^{2} q / a, e q / c, e q / d, b(q / a)^{\frac{1}{2}},-b(q / a)^{\frac{1}{2}} ; q,-\frac{a e q}{b c d}\right],
\end{gathered}
$$

provided $|a e q / b c d|<1$. Now if we specialize (5.13) by letting $d \rightarrow a / c$ and $e \rightarrow c$, the ${ }_{8} \phi_{7}$ series on the right hand side can be evaluated by the $q$-Watson summation in (2.4) and we obtain the following bilateral quadratic summation formula:

Corollary 5.6 Let $a, b$, and $c$ be indeterminates. Then

$$
\begin{aligned}
& \sum_{n=-\infty}^{\infty} \frac{\left(1-a q^{2 n}\right)}{(1-a)} \frac{(b, c, a / c ; q)_{n}}{(a q / b, a q / c, c q ; q)_{n}} q^{\left(\begin{array}{c}
n \\
2
\end{array}\right)}\left(-\frac{a q}{b}\right)^{n} \\
& \quad \times\left(b q^{1+n}, b q^{1-n} / a, c^{2} q^{2+n} / b, c^{2} q^{2-n} / a b ; q^{2}\right)_{\infty} \\
& =\frac{(q, q, a q, q / a, a q / b c, c q / b ; q)_{\infty}}{(q / b, a q / b, c q, q / c, a q / c, c q / a ; q)_{\infty}}\left(b q / c, b c q / a, c q^{2} / b, c^{3} q^{2} / b ; q^{2}\right)_{\infty}
\end{aligned}
$$

provided $|c q / b|<1$.

\subsection{A consequence of Theorem 4.3}

For illustration, we combine Theorem 4.3 with Shukla's very-well-poised ${ }_{8} \psi_{8}$ summation. We multiply both sides of (4.8) (which is equivalent to (4.9)) by

$$
\frac{\left(1-a q^{2 k}\right)}{(1-a)} \frac{\left(a / c, b, f, g, v, a q^{2} / v ; q\right)_{k}}{(c q, a q / b, a q / g, a q / g, a q / v, v / q ; q)_{k}}\left(\frac{a c}{b f g}\right)^{k}
$$

and sum over all integers $k$. On the right hand side we obtain

$$
\begin{aligned}
& \frac{(1-u / q)(1-a q / u)(q, a q / d, a q / e, q / d, q / e, c q / b, a q / b c, b c q / a, a b / c d e ; q)_{\infty}}{(b-c)(a / c d, a / c e, b q / d, b q / e, q / b, a q / b, c q, c q / a, a q / d e ; q)_{\infty}} \\
& \quad \times{ }_{10} \psi_{10}\left[\begin{array}{c}
q \sqrt{a},-q \sqrt{a}, d, e, f, g, u, a q^{2} / u, v, a q^{2} / v \\
\sqrt{a},-\sqrt{a}, a q / d, a q / e, a q / f, a q / g, a q / u, u / q, a q / v, v / q
\end{array} ; q, \frac{a^{2}}{\operatorname{def} g q}\right] .
\end{aligned}
$$

On the left hand side we obtain

$$
\begin{aligned}
& \sum_{k=-\infty}^{\infty} \frac{\left(1-a q^{2 k}\right)}{(1-a)} \frac{\left(a / c, b, f, g, v, a q^{2} / v ; q\right)_{k}}{(c q, a q / b, a q / g, a q / g, a q / v, v / q ; q)_{k}}\left(\frac{a c}{b f g}\right)^{k} \\
& \times \sum_{n=-\infty}^{\infty} \frac{\left(1-b c q^{2 n} / a\right)}{(1-b c / a)} \frac{\left(b q^{k}, b q^{-k} / a, c d q / a, c e q / a ; q\right)_{n}}{\left(c q^{1-k} / a, c q^{1+k}, b q / d, b q / e ; q\right)_{n}}\left(\frac{a}{d e}\right)^{n}
\end{aligned}
$$




$$
\begin{aligned}
& \times\left(1-\frac{(1-c d e / a b)\left(1-c q^{n+1} / u\right)\left(1-c u q^{n-1} / a\right)}{(1-c / b)\left(1-c d q^{n} / a\right)\left(1-c e q^{n} / a\right)}\right) \\
& =\sum_{n=-\infty}^{\infty} \frac{\left(1-b c q^{2 n} / a\right)}{(1-b c / a)} \frac{(b, b / a, c d q / a, c e q / a ; q)_{n}}{(c q / a, c q, b q / d, b q / e ; q)_{n}}\left(\frac{a}{d e}\right)^{n} \\
& \times\left(1-\frac{(1-c d e / a b)\left(1-c q^{n+1} / u\right)\left(1-c u q^{n-1} / a\right)}{(1-c / b)\left(1-c d q^{n} / a\right)\left(1-c e q^{n} / a\right)}\right) \\
& \times \sum_{k=-\infty}^{\infty} \frac{\left(1-a q^{2 k}\right)}{(1-a)} \frac{\left(a q^{-n} / c, b q^{n}, f, g, v, a q^{2} / v ; q\right)_{k}}{\left(c q^{1+n}, a q^{1-n} / b, a q / g, a q / g, a q / v, v / q ; q\right)_{k}}\left(\frac{a c}{b f g}\right)^{k}
\end{aligned}
$$

Now the inner sum, provided $|a c / b f g|<1$, can be evaluated by Shukla's very-well-poised ${ }_{8} \psi_{8}$ summation $(2.5)$ and we obtain

$$
\begin{gathered}
\sum_{n=-\infty}^{\infty} \frac{\left(1-b c q^{2 n} / a\right)}{(1-b c / a)} \frac{(b, b / a, c d q / a, c e q / a ; q)_{n}}{(c q / a, c q, b q / d, b q / e ; q)_{n}}\left(\frac{a}{d e}\right)^{n} \\
\times\left(1-\frac{(1-c d e / a b)\left(1-c q^{n+1} / u\right)\left(1-c u q^{n-1} / a\right)}{(1-c / b)\left(1-c d q^{n} / a\right)\left(1-c e q^{n} / a\right)}\right) \\
\times\left(1-\frac{(1-b / c)\left(1-b f q^{n} / a\right)\left(1-b g q^{n} / a\right)}{\left(1-b q^{1+n} / v\right)\left(1-b v q^{n-1} / a\right)(1-b f g / a c)}\right) \frac{\left(1-v q^{-1-n} / b\right)\left(1-b v q^{n-1} / a\right)}{(1-v / a q)(1-v / q)} \\
\times \frac{\left(q, a q, q / a, c q / b, a q^{1-n} / b f, a q^{1-n} / b g, c q^{1+n} / f, c q^{1+n} / g, a q / f g ; q\right)_{\infty}}{\left(a q^{1-n} / b, c q^{1+n}, a q / f, a q / g, q^{1-n} / b, c q^{1+n} / a, q / f, q / g, a c q / b f g ; q\right)_{\infty}} \\
=\frac{b v(q, a q, q / a, c q / b, a q / b f, a q / b g, c q / f, c q / g, a q / f g ; q)_{\infty}}{a q(1-v / a q)(1-v / q)(a q / b, c q, a q / f, a q / g, q / b, c q / a, q / f, q / g, a c q / b f g ; q)_{\infty}} \\
\times \sum_{n=-\infty}^{\infty} \frac{\left(1-b c q^{2 n} / a\right)}{(1-b c / a)} \frac{(c d q / a, c e q / a, b f q / a, b g q / a ; q)_{n}}{(b q / d, b q / e, c q / f, c q / g ; q)_{n}}\left(\frac{a}{d e q}\right)^{n} \\
\times\left(1-\frac{(1-c d e / a b)\left(1-c q^{n+1} / u\right)\left(1-c u q^{n-1} / a\right)}{(1-c / b)\left(1-c d q^{n} / a\right)\left(1-c e q^{n} / a\right)}\right) \\
\times\left(1-\frac{\left(1-b q^{1+n} / v\right)\left(1-b v q^{n-1} / a\right)(1-b f g / a c)}{(1-b / c)\left(1-b f q^{n} / a\right)\left(1-b g q^{n} / a\right)}\right) .
\end{gathered}
$$

Equating the last expression with (5.15), we obtain the following transformation for a particular very-well-poised ${ }_{10} \psi_{10}$ series:

Corollary 5.7 Let $a, b, c, d, e, f, g, u$, and $v$ be indeterminates. Then

$$
\begin{gathered}
{ }_{10} \psi_{10}\left[\begin{array}{c}
q \sqrt{a},-q \sqrt{a}, d, e, f, g, u, a q^{2} / u, v, a q^{2} / v \\
\sqrt{a},-\sqrt{a}, a q / d, a q / e, a q / f, a q / g, a q / u, u / q, a q / v, v / q
\end{array} ;, \frac{a^{2}}{d e f g q}\right] \\
=\frac{(b-c)(c-b)}{(1-u / q)(1-a q / u)(1-v / q)(1-a q / v)} \\
\times \frac{(a q, q / a, a / c d, a / c e, a q / d e, a q / b f, a q / b g, a q / f g, b q / d, b q / e, c q / f, c q / g ; q)_{\infty}}{(q / d, a q / d, q / e, a q / e, q / f, a q / f, q / g, a q / g, a q / b c, b c q / a, a b / c d e, a c q / b f g ; q)_{\infty}}
\end{gathered}
$$




$$
\begin{gathered}
\times \sum_{n=-\infty}^{\infty} \frac{\left(1-b c q^{2 n} / a\right)}{(1-b c / a)} \frac{(c d q / a, c e q / a, b f q / a, b g q / a ; q)_{n}}{(b q / d, b q / e, c q / f, c q / g ; q)_{n}}\left(\frac{a}{d e q}\right)^{n} \\
\times\left(1-\frac{(1-c d e / a b)\left(1-c q^{n+1} / u\right)\left(1-c u q^{n-1} / a\right)}{(1-c / b)\left(1-c d q^{n} / a\right)\left(1-c e q^{n} / a\right)}\right) \\
\quad \times\left(1-\frac{\left(1-b q^{1+n} / v\right)\left(1-b v q^{n-1} / a\right)(1-b f g / a c)}{(1-b / c)\left(1-b f q^{n} / a\right)\left(1-b g q^{n} / a\right)}\right),
\end{gathered}
$$

provided $\mid a^{2} /$ def $g q \mid<1$ and $\mid a /$ deq $\mid<1$.

As usual, we have applied analytic continuation to establish the conditions of convergence.

We could also combine Theorem 4.3 with (4.10) or with other identities. With Corollary 5.7, we just wanted to provide one example from the many possibilities of deducing a transformation from Theorem 4.3.

\section{References}

[1] A. K. Agarwal, G. E. Andrews, and D. M. Bressoud, "The Bailey lattice", J. Indian Math. Soc. (N. S.) 51 (1987), 57-73.

[2] K. Alladi, G. E. Andrews, and A. Berkovich, "A new four parameter $q$-series identity and its partition implications", Inv. Math., to appear.

[3] W. A. Al-Salam and A. Verma, "On quadratic transformations of basic series", SIAM J. Math. Anal. 15 (2) (1984), 414-420.

[4] G. E. Andrews, "Applications of basic hypergeometric functions", SIAM Rev. 16 (1974), 441-484.

[5] G. E. Andrews, "Connection coefficient problems and partitions", D. Ray-Chaudhuri, ed., Proc. Symp. Pure Math. 34, (Amer. Math. Soc., Providence, RI, 1979), 1-24.

[6] G. E. Andrews, q-Series: Their development and application in analysis, number theory, combinatorics, physics and computer algebra, CBMS Regional Conference Lectures Series 66 (Amer. Math. Soc., Providence, RI, 1986).

[7] G. E. Andrews, "Bailey's transform, lemma, chains and tree", in Special Fuctions 2000: Current perspective and future directions (J. Bustoz, M. E. H. Ismail, S. K. Suslov, eds.), NATO Sci. Ser. II: Math. Phys. Chem. 30 (2001), 1-22.

[8] G. E. Andrews, R. Askey and R. Roy, Special functions, Encyclopedia of Mathematics And Its Applications 71, Cambridge University Press, Cambridge (1999).

[9] R. Askey and M. E. H. Ismail, "The very well poised ${ }_{6} \psi_{6}$ ", Proc. Amer. Math. Soc. 77 (1979), 218-222. 
[10] W. N. Bailey, "Series of hypergeometric type which are infinite in both directions", Quart. J. Math. (Oxford) 7 (1936), 105-115.

[11] W. N. Bailey, "Well-poised basic hypergeometric series", Quart. J. Math. (Oxford) 18 (1947), 157-166.

[12] G. Bhatnagar and S. C. Milne, "Generalized bibasic hypergeometric series and their U(n) extensions", Adv. Math. 131 (1997), 188-252.

[13] D. M. Bressoud, "A matrix inverse", Proc. Amer. Math. Soc. 88 (1983), 446-448.

[14] L. Carlitz, "Some inverse relations", Duke Math. J. 40 (1973), 893-901.

[15] W.-C. Chu, "Some multifold reciprocal transformations with applications to series expansions", Europ. J. Comb. 12 (1991), 1-8.

[16] W.-C. Chu, "Partial fraction expansions and well-poised bilateral series", Acta Sci. Math. (Szeged) 64 (1998), 495-513.

[17] G. Gasper, "Summation, transformation and expansion formulas for bibasic series", Trans. Amer. Soc. 312 (1989), 257-278.

[18] G. Gasper and M. Rahman, "An indefinite bibasic summation formula and some quadratic, cubic and quartic summation and transformation formulae", Canad. J. Math. 42 (1990), 1-27.

[19] G. Gasper and M. Rahman, Basic hypergeometric series, Encyclopedia of Mathematics And Its Applications 35, Cambridge University Press, Cambridge (1990).

[20] I. Gessel and D. Stanton, "Application of $q$-Lagrange inversion to basic hypergeometric series", Trans. Amer. Math. Soc. 277 (1983), 173-203.

[21] H. W. Gould and L. C. Hsu, "Some new inverse series relations", Duke Math. J. 40 (1973), 885-891.

[22] R. A. Gustafson, "The Macdonald identities for affine root systems of classical type and hypergeometric series very well-poised on semi-simple Lie algebras", in Ramanujan International Symposium on Analysis (Dec. 26th to 28th, 1987, Pune, India), N. K. Thakare (ed.) (1989), 187-224.

[23] M. E. H. Ismail and D. R. Masson, " $q$-Hermite polynomials, biorthogonal rational functions, and q-beta integrals", Trans. Amer. Math. Soc. 346 (1994), 63-116.

[24] F. H. Jackson, "Summation of $q$-hypergeometric series", Messenger of Math. 57 (1921), 101-112.

[25] M. Jackson, "On well-poised bilateral hypergeometric series of type ${ }_{8} \psi_{8}$ ", Quart. J. Math. (Oxford) (2) 1 (1950), 63-68. 
[26] C. Krattenthaler, "A new matrix inverse", Proc. Amer. Math. Soc. 124 (1996), 4759.

[27] C. Krattenthaler and M. Schlosser, "A new multidimensional matrix inverse with applications to multiple q-series", Discrete Math. 204 (1999), 249-279.

[28] G. M. Lilly and S. C. Milne, "The $C_{l}$ Bailey Transform and Bailey Lemma", Constr. Approx. 9 (1993), 473-500.

[29] S. C. Milne, "A multiple series transformation of the very well poised ${ }_{2 k+4} \Psi_{2 k+4}$ ", Pacif. J. Math. 91 (2) (1980), 419-430.

[30] S. C. Milne, "Balanced ${ }_{3} \phi_{2}$ summation theorems for $U(n)$ basic hypergeometric series", Adv. Math. 131 (1997), 93-187.

[31] J. Riordan, Combinatorial identities, J. Wiley, New York, 1968.

[32] L. J. Rogers, "Third memoir on the expansion of certain infinite products", Proc. London Math. Soc. 26 (1894), 15-32.

[33] H. Rosengren, "Reduction formulae for Karlsson-Minton type hypergeometric functions", Constr. Approx., to appear.

[34] H. Rosengren, "Karlsson-Minton type hypergeometric functions on the root system $C_{n} "$, J. Math. Anal. Appl., to appear.

[35] M. Schlosser, "Multidimensional matrix inversions and $A_{r}$ and $D_{r}$ basic hypergeometric series", The Ramanujan J. 1 (1997), 243-274.

[36] M. Schlosser, "A new multidimensional matrix inversion in $A_{r}$ ", Contemp. Math. 254 (2000), 413-432.

[37] M. Schlosser, "Multilateral transformations of $q$-series with quotients of parameters that are nonnegative integral powers of $q$ ", in $q$-Series with Applications to Combinatorics, Number Theory, and Physics (B. C. Berndt and K. Ono, eds.), Amer. Math. Soc., Providence, R. I., Contemp. Math. 291 (2001), 203-227.

[38] M. Schlosser, "A simple proof of Bailey's very-well-poised ${ }_{6} \psi_{6}$ summation", Proc. Amer. Math. Soc. 130 (2002), 1103-1111.

[39] M. Schlosser, "Elementary derivations of identities for bilateral basic hypergeometric series", Selecta Math. (N. S.), to appear.

[40] H. S. Shukla, "A note on the sums of certain bilateral hypergeometric series", Proc. Cambridge Phil. Soc. 55 (1959), 262-266.

[41] S. O. Warnaar, "Summation and transformation formulas for elliptic hypergeometric series", Constr. Approx. 18 (2002), 479-502. 
[42] G. N. Watson, "A note on generalized hypergeometric series", Proc. London Math. Soc. (2) 23 (1925), xiii-xv.

[43] F. J. W. Whipple, "A group of generalized hypergeometric series: relations between 120 allied series of the type $F[a, b, c ; d, e] "$, Proc. London Math. Soc. (2) 23 (1925), $104-114$.

[44] E. T. Whittaker and G. N. Watson, A course of modern analysis, 4th ed., Cambridge University Press, Cambridge, 1962. 